\title{
The submillimeter C and CO lines in Henize 2-10 and NGC 253
}

\author{
E. Bayet ${ }^{1}$, M. Gerin ${ }^{1}$, T. G. Phillips ${ }^{2}$, and A. Contursi ${ }^{3}$ \\ ${ }^{1}$ Laboratoire de Radioastronomie (LRA), Observatoire de Paris and École Normale Supérieure, 24 rue Lhomond, \\ 75005 Paris, France (CNRS-UMR 8112) \\ e-mail: estelle.bayet@lra.ens.fr \\ 2 California Institute of Technology, Downs Laboratory of Physics 320-47, Pasadena, CA 91125, USA \\ ${ }^{3}$ Max Planck Institute für Extraterrestrische Physik, Postfach 1312, 85741, Garching, Germany
}

Received 3 November 2003 / Accepted 5 July 2004

\begin{abstract}
The purpose of this paper is to describe a method for determining a cooling template for galaxies, using nearby galaxies, and applicable to future observations of distant galaxies. We observed two starburst galaxies (NGC 253 and Henize 2-10) with the Caltech Submillimeter Observatory in the rotational lines of carbon monoxide ${ }^{12} \mathrm{CO}(J=3-2),(J=6-5)$ and $(J=7-6)$ for both, and also ${ }^{12} \mathrm{CO}(J=4-3)$ and ${ }^{13} \mathrm{CO}(J=3-2)$ for Henize 2-10 and in the ${ }^{3} \mathrm{P}_{2}-{ }^{3} \mathrm{P}_{1}$ fine-structure transitions of atomic carbon $[\mathrm{CI}]$ at $809 \mathrm{GHz}$ for NGC 253. Some of these observations have been made previously, but the present multitransition study (including data found in the literature) is the most complete to date for the two galaxies. From these observations, we have derived the properties of the warm and dense molecular gas in the galaxy nuclei. We used an LTE analysis and an LVG radiative transfer model to determine physical conditions of the interstellar medium in both sources and predicted integrated line properties of all $\mathrm{CO}$ transitions up to ${ }^{12} \mathrm{CO}(15-14)$. We found the observations to be in good agreement with a medium characterized by $T_{\mathrm{k}} \approx 50-100 \mathrm{~K}, \frac{{ }^{12} \mathrm{CO}}{1{ }^{3} \mathrm{CO}} \approx 30, n\left(\mathrm{H}_{2}\right) \approx 10^{4} \mathrm{~cm}^{-3}$ and $N\left({ }^{12} \mathrm{CO}\right)=3.5 \pm 1 \times 10^{18} \mathrm{~cm}^{-2}$ for Henize 2-10 and characterized by $T_{\mathrm{k}} \approx 70-150 \mathrm{~K}, \frac{{ }^{12} \mathrm{CO}}{{ }^{13} \mathrm{CO}} \approx 40, n\left(\mathrm{H}_{2}\right) \approx 10^{4} \mathrm{~cm}^{-3}$ and $N\left({ }^{12} \mathrm{CO}\right)=1.5 \pm 0.5 \times 10^{19} \mathrm{~cm}^{-2}$ for NGC 253 . A PDR model has also been used and here the data are well fitted (within $20 \%$ ) by a model cloud with a gas density of $n(\mathrm{H})=8.0 \pm 1 \times 10^{5} \mathrm{~cm}^{-3}$ and an incident FUV flux of $\chi \approx 20000$ for Henize 2-10. For NGC 253, we deduced $n(\mathrm{H})=3.0 \pm 0.5 \times 10^{5} \mathrm{~cm}^{-3}$ and $\chi \approx 20000$ for the modelled cloud. The physical properties of warm gas and CO cooling curves of the target galaxies are compared with those measured for the nucleus of the Milky Way and the Cloverleaf QSO. The gas properties and CO cooling curve are similar for the two starburst galaxies and the Cloverleaf QSO while the Milky Way nucleus exhibits lower excitation molecular gas.
\end{abstract}

Key words. galaxies: starburst - galaxies: individual: NGC 253 - galaxies: individual: Henize 2-10 - submillimeter ISM: molecules - galaxies: ISM

\section{Introduction}

Over the past decade, observations of distant galaxies have become possible in the millimeter and submillimeter bands and considerable progress is expected for the next decade. Much of the energy provided by star formation in those galaxies is down-converted to the submm and appears as dust emission and the familiar gas cooling lines. Because the information on distant galaxies is scarce, due to the lack of resolution and sensitivity, it is essential to understand nearby galaxies in order to provide templates for more distant objects. In recent studies of emission of fine-structure transitions of atomic carbon [CI], ionized atomic carbon [CII] and carbon monoxide (CO), we have begun to see how they relate to each other and to dust emission (Gerin \& Phillips 1998, 2000). However, to estimate the relative contributions of the various species to the gas cooling, it is often necessary to make assumptions for the power in the various unobserved lines.

It is well known that the fine-structure lines of ionized carbon $[\mathrm{CII}]$ and atomic oxygen oxygen [OI] contribute most of the cooling of the neutral interstellar gas in galaxies. These lines trace the cooling of the diffuse neutral gas with a significant contribution from Photo-Dissociation Regions (PDRs). Fine-structure lines of ionized oxygen [OIII] and nitrogen [NII] trace the ionized gas, either in HII regions ([OIII]) or the diffuse ionised gas ([NII]). In molecular gas, the cooling radiation is due to atomic carbon, carbon monoxide and water lines (although water (like most molecules) is not sufficiently widespread to contribute much cooling to a galaxy as a whole). These predictions have been confirmed by the COBE-FIRAS observations of the Milky Way: apart from [CII] and [NII] and probably [OI], the most intense submillimeter lines are from C and CO (see Fixsen et al. 1999 and Sect. 4.2). The relative contributions of the different lines of $\mathrm{C}$ and $\mathrm{CO}$ vary along the Galactic plane. Also C contributes less in proportion to the total cooling towards the Galactic Center than towards the rest of the disk (Bennett et al. 1994 and Fixsen et al. 1999).

The detection of $\mathrm{CO}$ lines of distant galaxies remains an extremely difficult task and will continue so until ALMA is 
ready. However, detecting their submillimeter continuum emission is now feasible, with the advent of sensitive bolometer arrays. Due to the negative K-correction effect, the dust emission of distant galaxies piles up at submillimeter wavelengths irrespective of redshift. Additional information is needed to be able to estimate a photometric redshift, or to classify the galaxies. For instance, Yun \& Carilli (2002) have proposed to use the $\mathrm{cm}$ radio continuum emission for obtaining photometric redshifts. From the model developed by Lagache et al. (2003) to explain the evolution of number counts in the submillimeter and far infrared (FIR), it appears that two main galaxy populations contribute to these number counts: a relatively local population of "cold" galaxies, similar to our own galaxy, in which the continuum emission is dominated by extended disktype emission, and an additional, strongly evolving population of infrared (IR) luminous galaxies, similar to local starburst galaxies. From the information gathered so far on high- $J$ CO emission in galaxies (this work; Israel \& Baas 2002, 2003; Bradford et al. 2003; Ward et al. 2003, and references therein), it is likely that high- $J$ CO lines $\left({ }^{12} \mathrm{CO}(4-3)\right.$ and above) will be more prominent in actively star forming galaxies compared to the less active galaxies in which only low- $J$ CO lines and the carbon lines are expected. In this paper, from an extensive set of observations of submillimeter CO lines, we conclude that, relative to ${ }^{12} \mathrm{CO}(1-0)$, starburst galaxies do exhibit stronger high-excitation $\mathrm{CO}$ lines $\left({ }^{12} \mathrm{CO}(4-3)\right.$ and above), than the Milky Way galaxy.

Determining the physical conditions of molecular clouds in external galaxies can be difficult. For single-dish observations of external galaxies, the beam size is usually larger than the angular diameter of typical molecular clouds, hence the cloud emission is beam-diluted. In addition, molecular clouds are known to be clumpy (e.g. Stutzki \& Guesten 1990). The intensities of $\mathrm{CO}$ rotational lines are sensitive to the gas physical conditions $\left(\mathrm{H}_{2}\right.$ density $n\left(\mathrm{H}_{2}\right)$, and kinetic temperature $\left.T_{\mathrm{k}}\right)$, the ${ }^{12} \mathrm{CO}$ column density $N\left({ }^{12} \mathrm{CO}\right)$ as well as the velocity field. In this paper, we use several methods for determining the ${ }^{12} \mathrm{CO}$ column density and the physical conditions, and for predicting the intensity of all $\mathrm{CO}$ rotational lines up to ${ }^{12} \mathrm{CO}(15-14)$. The LTE analysis is useful for constraining the kinetic temperature. Using LVG models, three parameters $\left(n\left(\mathrm{H}_{2}\right), T_{\mathrm{k}}, N\left({ }^{12} \mathrm{CO}\right)\right)$ can be measured. PDR models include a self consistent treatment of the thermal and chemical processes and provide therefore a better theoretical understanding of the gas properties.

We recall that, assuming LTE, the brightness temperature, $T_{\mathrm{B}}$, of CO lines as a function of the kinetic temperature $T_{\mathrm{k}}$ can be written as:

$T_{\mathrm{B}}=F F\left(J_{v}\left(T_{\mathrm{k}}\right)-J_{v}\left(T_{\mathrm{bg}}\right)\right)\left(1-\mathrm{e}^{-\tau}\right)$

where $F F$ is the source surface filling factor in the beam, $\tau$ is the line opacity, $T_{\mathrm{bg}}$ is the CMB temperature $\left(T_{\mathrm{bg}}=2.7 \mathrm{~K}\right)$ and $J_{v}\left(T_{\mathrm{k}}\right)$ is described in Kutner \& Ulich (1981) by:

$J_{v}\left(T_{\mathrm{k}}\right)=\left(\frac{h v}{k_{\mathrm{b}}}\right) \times \frac{1}{\exp \left(\frac{h v}{k_{\mathrm{b}} T_{\mathrm{k}}}\right)-1}$

where $k_{\mathrm{b}}$ is the Boltzmann's constant and $h$ is the Planck constant. We show in Fig. 1 the brightness temperature, $T_{\mathrm{B}}$, as a

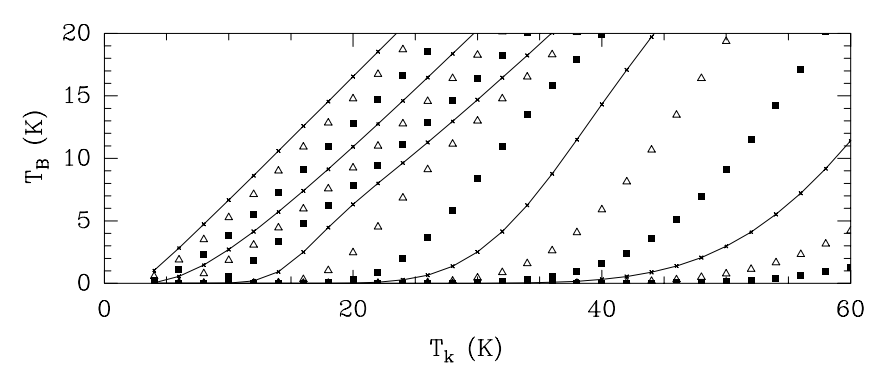

Fig. 1. Brightness temperature $T_{\mathrm{B}}$ (Eqs. (1) and (2) in Sect. 1) vs. the kinetic temperature, $T_{\mathrm{k}}$, assuming a unity filling factor $(F F=$ 1). The first curve on the left is ${ }^{12} \mathrm{CO}(1-0)$ (full line) followed by ${ }^{12} \mathrm{CO}(2-1)$ (triangles), ${ }^{12} \mathrm{CO}(3-2)$ (squares), ${ }^{12} \mathrm{CO}(4-3)$ (full line)... up to ${ }^{12} \mathrm{CO}(15-14)$. For this plot, we used $N(\mathrm{CO})=1 \times 10^{18} \mathrm{~cm}^{-2}$ and $\Delta v=1 \mathrm{~km} \mathrm{~s}^{-1}$ resulting in $\tau \gg 1$.

function of the kinetic temperature $T_{\mathrm{k}}$. In this example, typical figures for Galactic molecular clouds have been chosen with $F F=1, N\left({ }^{12} \mathrm{CO}\right)=1 \times 10^{18} \mathrm{~cm}^{-2}$ and $\Delta v=1 \mathrm{~km} \mathrm{~s}^{-1}$, resulting in saturated $\mathrm{CO}$ lines.

For a gas temperature around $T_{\mathrm{k}} \approx 10 \mathrm{~K}$, low- $J$ CO lines $\left({ }^{12} \mathrm{CO}(1-0),{ }^{12} \mathrm{CO}(2-1)\right.$ and $\left.{ }^{12} \mathrm{CO}(3-2)\right)$ are easily detected while mid and high- $J \mathrm{CO}$ lines $\left({ }^{12} \mathrm{CO}(5-4),{ }^{12} \mathrm{CO}(6-5)\right.$ and above) need warmer gas to be detected $\left(T_{\mathrm{k}} \gtrsim 15 \mathrm{~K}\right)$.

Since we want to sample warm gas, we have observed, for both galaxies, the high- $J$ CO lines: ${ }^{12} \mathrm{CO}(4-3)$ (only for Henize 2-10), ${ }^{12} \mathrm{CO}(6-5)$ and ${ }^{12} \mathrm{CO}(7-6)$ which are more sensitive to warm, dense gas directly involved in the starburst. We also observed ${ }^{12} \mathrm{CO}(3-2)$ for both galaxies and ${ }^{13} \mathrm{CO}(3-2)$ for Henize 2-10, to better constrain the models (see Sect. 5.1).

Atomic carbon has also proved to be a good tracer of molecular gas (Israel \& Baas 2001; Gerin \& Phillips 2000). The fine structure transition of atomic carbon ${ }^{3} \mathrm{P}_{2}-{ }^{3} \mathrm{P}_{1}[\mathrm{CI}]$ at $809 \mathrm{GHz}$ for NGC 253 has also been observed here, since the intensity ratio between this line and the ${ }^{3} \mathrm{P}_{1}-{ }^{3} \mathrm{P}_{0}[\mathrm{CI}]$ at $492 \mathrm{GHz}$ is a sensitive tracer of the total gas pressure. For NGC 253, Bradford et al. (2003) and Israel et al. (1995) give the intensity of the fine structure transition of atomic carbon ${ }^{3} \mathrm{P}_{1}-{ }^{3} \mathrm{P}_{0}[\mathrm{CI}]$ at $492 \mathrm{GHz}$. For Henize 2-10, Gerin \& Phillips (2000) give the intensity of the ${ }^{3} \mathrm{P}_{1}-{ }^{3} \mathrm{P}_{0}[\mathrm{CI}]$ at $492 \mathrm{GHz}$ but we could not find a measure of ${ }^{3} \mathrm{P}_{2}-{ }^{3} \mathrm{P}_{1}[\mathrm{CI}]$ at $809 \mathrm{GHz}$ to compute the line ratio.

In the following, we present new $\mathrm{CO}$ and $\mathrm{CI}$ data for NGC 253 and for Henize 2-10 (see Sect. 3). The galaxies are described in Sect. 2, the observations in Sect. 3. The results and data analysis providing ISM properties are discussed in Sects. 4 and 5, respectively, with conclusions in Sect. 6.

\section{The sample}

Henize 2-10 is a blue compact dwarf galaxy with a metallicity of $12+\log \left(\frac{\mathrm{O}}{\mathrm{H}}\right) \approx 8.93$ (Zaritsky et al. 1994). It presents a double core structure suggesting a galaxy merger (Baas et al. 1994). From optical, infrared and radio observations of Henize $2-10$, the derived distance is $6 \mathrm{Mpc}$ (Johansson 1987). Henize 2-10 is rich in neutral atomic hydrogen (Allen et al. 1976) and harbors a considerable 
population of Wolf-Rayet stars (D'Odorico et al. 1983; Kawara et al. 1987). Previous CO observations have been reported by Baas et al. (1994); Kobulnicky et al. (1995, 1999) and Meier et al. (2001). The starburst is confined in the galaxy nucleus within a radius of $5^{\prime \prime}(150 \mathrm{pc})$. This galaxy was chosen because it shows intense and narrow CO lines.

NGC 253 is a highly inclined galaxy $\left(i=78^{\circ}\right.$, Pence 1980) of type Sc. With M 82, it is the best nearby example of a nuclear starburst (Rieke et al. 1988). Blecha (1986) observed 24 of the brightest globular clusters and estimated a distance of 2.4 to $3.4 \mathrm{Mpc}$, while Davidge \& Pritchet (1990) analysed a color-magnitude diagram of stars in the halo of NGC 253 and conclude that the distance is 1.7 to $2.6 \mathrm{Mpc}$. In the following, we shall use a value of $D=2.5 \mathrm{Mpc}$ as in Mauersberger et al. (1996). NGC 253 has been extensively observed at all wavelengths (Turner 1985 (map in all four $18 \mathrm{~cm}$ lines of $\mathrm{OH}$ in the nucleus); Antonucci \& Ulvestad 1988 (deep VLA maps of NGC 253 which show at least 35 compact radio sources); Carlstrom et al. 1990 (the $J=1-0$ transitions of HCN and $\mathrm{HCO}^{+}$and the $3 \mathrm{~mm}$ continuum emission); Telesco \& Harper 1980 (30 and $300 \mu \mathrm{m}$ emission); Strickland et al. 2004 (X-ray observations)). NGC 253 presents a very active and young starburst. It is among the first galaxies to have been detected in submillimeter lines and continuum. The NGC $253 \mathrm{nu}-$ cleus has been mapped previously in various lines of $\mathrm{CO}$ and C (Bradford et al. 2003; Israel \& Baas 2002; Sorai et al. 2000; Harrison et al. 1999; Israel et al. 1995; Wall et al. 1991; Harris et al. 1991). The most intense emission is associated with the starburst nucleus with additional weak and extended emission from the disk (Sorai et al. 2000). Both galaxies harbor superstar clusters in their nuclei (Keto et al. 1999) similar to the superstar clusters found in interacting galaxies (Mirabel et al. 1998). Basic properties for both galaxies are summarized in Table 1.

\section{Observations}

The observations were made during various sessions at the Caltech Submillimeter Observatory (CSO) in Hawaii (USA) with the Superconducting Tunnel Junction receivers operated in double-side band mode. The atmospheric conditions varied from good $\left(\tau_{225} \lesssim 0.1\right)$ to excellent $\left(\tau_{225} \approx 0.06\right)$. We used a chopping secondary mirror with a throw of 1 to 3 arcmin, depending on the size of the source, and with a frequency around $1 \mathrm{~Hz}$. Henize 2-10 is point-like for all lines. The NGC 253 nucleus has a size of 50" (see Fig. 3) which is smaller than the chopping throw. For this galaxy, we used a $3^{\prime}$ chopping throw in ${ }^{12} \mathrm{CO}(3-2)$. There is no sign of contamination by emission in the off beams. We restricted the chopping throw to $1^{\prime}$ for ${ }^{12} \mathrm{CO}(6-5)$ and ${ }^{12} \mathrm{CO}(7-6)$ as the emission is very compact in these lines. Spectra were measured with two acousto-optic spectrometers (effective bandwidth of $1000 \mathrm{MHz}$ and $500 \mathrm{MHz}$ ). The first one has a spectral resolution about $1.5 \mathrm{MHz}$ and the second one about $2 \mathrm{MHz}$. The IF frequency of the CSO receivers is $1.5 \mathrm{GHz}$. The main beam efficiencies $(\eta)$ of the CSO were $69.8 \%, 74.6 \%, 51.5 \%, 28 \%$ and $28 \%$ at 230 , $345,460,691$ and $806 \mathrm{GHz}$ respectively, as measured on planets. We used the ratio $\frac{1}{\eta}$ to convert $T_{A}^{*}$ into $T_{\mathrm{mb}}$. The beam size at
Table 1. Basic properties of Henize 2-10 and NGC 253.

\begin{tabular}{ll}
\hline \hline Henize 2-10 & \\
\hline RA (1950) & $08: 34: 07.2$ \\
Dec (1950) & $-14: 26: 06.0$ \\
Distance & $6 \mathrm{Mpc}^{1}$ \\
Velocity (LSR) & $+850 \mathrm{~km} \mathrm{~s}^{-1}$ \\
Optical size & $30^{\prime \prime} \times 40^{\prime \prime}$ \\
Position angle & $130^{\circ}$ \\
Inclination & $44^{\circ}$ \\
Metallicity & $12+\log \left(\frac{\mathrm{O}}{\mathrm{H}}\right) \approx 8.93^{2}$ \\
$L_{\mathrm{FIR}}$ & $1.7 \times 10^{9} L_{\odot}^{3}$ \\
Absolute luminosity $L_{\mathrm{B}}$ & $1.2 \times 10^{9} L_{\odot}^{4}$ \\
\hline NGC 253 & \\
\hline RA (1950) & $00: 45: 05.7$ \\
Dec (1950) & $-25: 33: 38.0$ \\
Distance & $2.5 \mathrm{Mpc}^{5}$ \\
Velocity (LSR) & $+240 \mathrm{~km} \mathrm{~s}^{-1}$ \\
Optical size & $27.5^{\prime} \times 6.8^{\prime}$ \\
Position angle & $51^{\circ}$ \\
Inclination & $78.5^{\circ}$ \\
Metallicity & $12+\log \left(\frac{\mathrm{O}}{\mathrm{H}}\right)=8.99 \pm 0.31^{2}$ \\
$L_{\mathrm{FIR}}$ & $1.0 \times 10^{10} L_{\odot}^{3}$ \\
Absolute luminosity $L_{\mathrm{B}}$ & $2.7 \times 10^{10} L_{\odot}^{6}$ \\
\hline & \\
\hline
\end{tabular}

${ }^{1}$ Johansson (1987).

${ }^{2}$ Zaritsky et al. (1994).

${ }^{3}$ Computed from formula in Imanishi \& Dudley (2000) and from Sanders \& Mirabel (1996).

${ }^{4}$ Baas, Israel \& Koornneef (1994).

${ }^{5}$ As in Mauersberger et al. (1996) (see text).

${ }^{6}$ Pence (1980).

$230,345,460,691$ and $806 \mathrm{GHz}$ is $30.5^{\prime \prime}, 21.9^{\prime \prime}, 14.5^{\prime \prime}, 10.6^{\prime \prime}$ and $8.95^{\prime \prime}$ respectively ${ }^{1}$.

The pointing was checked using planets (Jupiter, Mars and Saturn) and evolved stars (IRC 10216 and R-Hya). The pointing accuracy is around $5^{\prime \prime}$. The overall calibration accuracy is $20 \%$. Data have been reduced using the CLASS data analysis package. The spectra have been smoothed to a velocity resolution of $10 \mathrm{~km} \mathrm{~s}^{-1}$ and linear baselines have been removed.

For NGC 253, we obtained spectra towards the central position for both ${ }^{12} \mathrm{CO}(7-6)$ line and the atomic carbon $\mathrm{CI}\left({ }^{3} \mathrm{P}_{2}-{ }^{3} \mathrm{P}_{1}\right)$ line simultaneously. We observed 70 positions in the ${ }^{12} \mathrm{CO}(3-2)$ line and 20 positions for the ${ }^{12} \mathrm{CO}(6-5)$ line. For Henize 2-10 we observed only the nucleus for the ${ }^{12} \mathrm{CO}(3-2)$, ${ }^{12} \mathrm{CO}(4-3),{ }^{12} \mathrm{CO}(6-5),{ }^{12} \mathrm{CO}(7-6)$ and ${ }^{13} \mathrm{CO}(3-2)$ lines. The carbon line ${ }^{3} \mathrm{P}_{1}-{ }^{3} \mathrm{P}_{0}[\mathrm{CI}]$ is from Gerin \& Phillips (2000).

\section{Data analysis}

\subsection{Spectra and maps}

Henize 2-10 and NGC 253 spectra at central positions are shown in Fig. 2; Fig. 3 shows ${ }^{12} \mathrm{CO}(3-2)$ and ${ }^{12} \mathrm{CO}(6-5)$ maps of NGC 253 . We detected ${ }^{12} \mathrm{CO}(3-2),{ }^{12} \mathrm{CO}(6-5)$ and

\footnotetext{
${ }^{1}$ See web site: http://www. submm. caltech.edu/cso/
} 

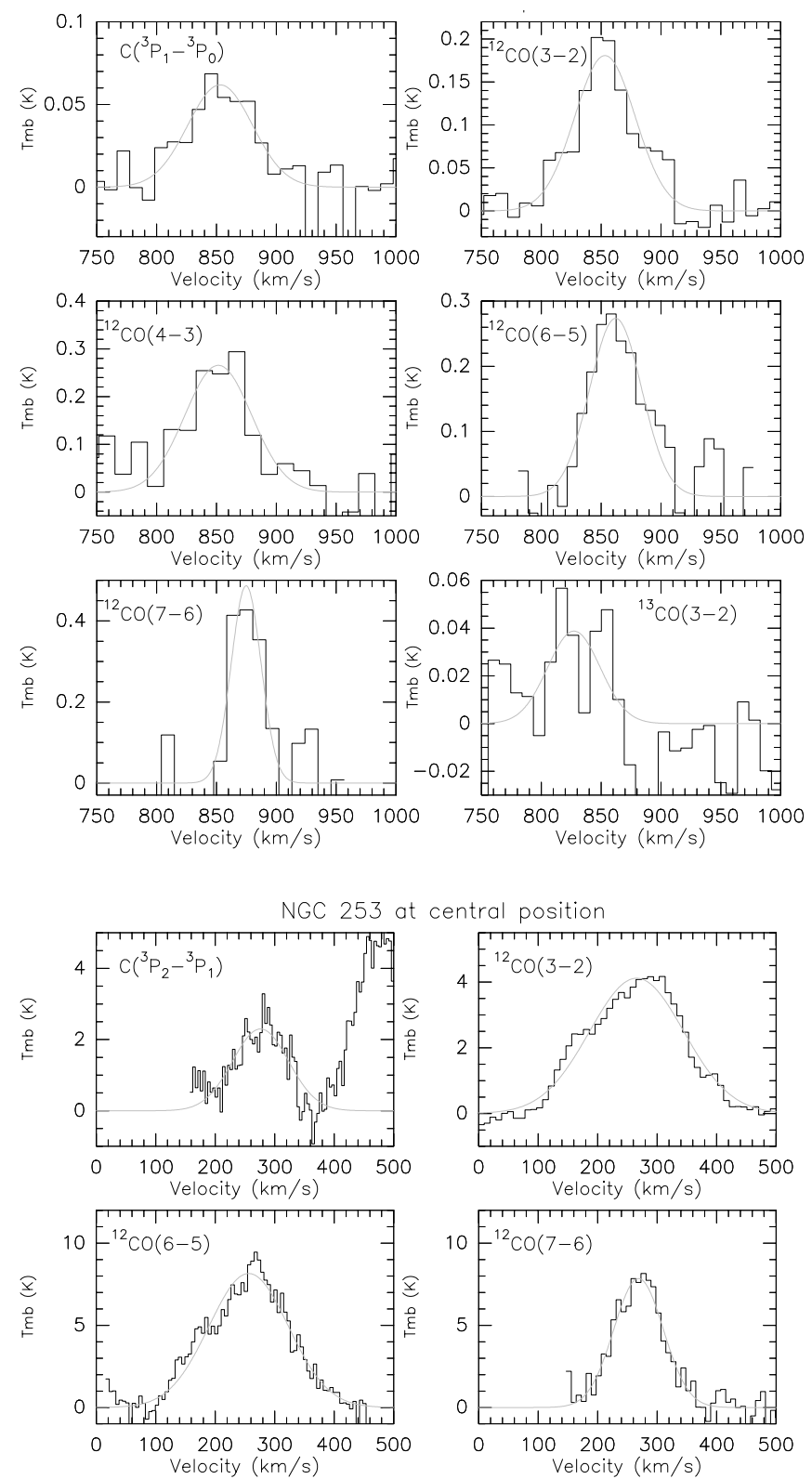

Fig. 2. Spectra of Henize 2-10 (top six) and NGC 253 (bottom four) at central positions as given in Table 1. Velocities (horizontal axis) are given relative to the LSR $\left(V_{\mathrm{LSR}}\right)$ in $\mathrm{km} \mathrm{s}^{-1}$ and the line intensities (vertical axis) are in units of $T_{\mathrm{mb}}(\mathrm{K})$. The grey curves are Gaussian fits. For NGC 253, the line on the right hand side of $\mathrm{CI}\left({ }^{3} \mathrm{P}_{2}-{ }^{3} \mathrm{P}_{1}\right)$ is the edge of ${ }^{12} \mathrm{CO}(7-6)$ line seen in the image side band.

${ }^{12} \mathrm{CO}(7-6)$ in both galaxies. For these positions, line intensities $\left(A\right.$ in $\mathrm{K} \mathrm{km} \mathrm{s}^{-1}$ and $I$ in $\mathrm{W} \mathrm{m}^{-2} \mathrm{sr}^{-1}$ ) and line fluxes $\left(F\right.$ in $\mathrm{W} \mathrm{m}^{-2}$ ) resulting from Gaussian fits are listed in Tables 2 and 3, together with the beam size of the observations. Because of likely pointing offsets $\left(\approx 5^{\prime \prime}\right.$ from pointing scans on planets and evolved stars) between different observing runs, we believe that differences in the peak positions of ${ }^{12} \mathrm{CO}(3-2)$ and ${ }^{12} \mathrm{CO}(6-5)$ in NGC 253 are partly due to pointing errors. But we can not exclude that a difference in peak positions remains after the pointing correction (see Fig. 3). Three peaks appear on the high resolution ${ }^{12} \mathrm{CO}(1-0)$ maps obtained with the

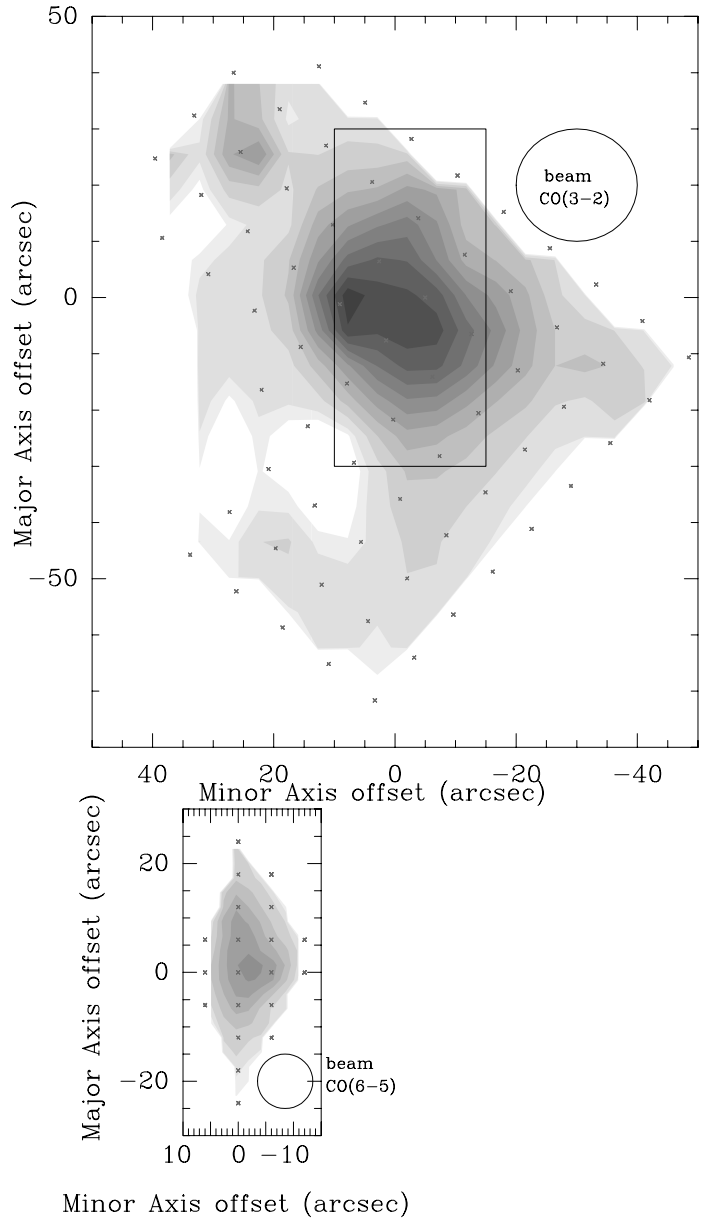

Fig. 3. Map of the velocity integrated intensity of ${ }^{12} \mathrm{CO}(3-2)$ (Top) and ${ }^{12} \mathrm{CO}(6-5)$ (Bottom) towards NGC 253 nucleus. The $\mathrm{CO}$ emission is integrated over the full velocity range -100 to $700 \mathrm{~km} \mathrm{~s}^{-1}$ and 60 to $400 \mathrm{~km} \mathrm{~s}^{-1}$, respectively. For both maps, missing grid positions are interpolated and the crosses show the observed positions. The first and the second contours of the ${ }^{12} \mathrm{CO}(3-2)$ map are of $41.6 \mathrm{~K} \mathrm{~km} \mathrm{~s}^{-1}$ and $81.6 \mathrm{~K} \mathrm{~km} \mathrm{~s}^{-1}$ respectively; others contours range from $\int T_{\mathrm{mb}} \mathrm{d} v=$ 81.6 to $816 \mathrm{~K} \mathrm{~km} \mathrm{~s}^{-1}$ in steps of $81.6 \mathrm{~K} \mathrm{~km} \mathrm{~s}^{-1}$. For the ${ }^{12} \mathrm{CO}(6-5)$ map, contours are of $43 \mathrm{~K} \mathrm{~km} \mathrm{~s}^{-1}, 141 \mathrm{~K} \mathrm{~km} \mathrm{~s}^{-1}$, $282 \mathrm{~K} \mathrm{~km} \mathrm{~s}^{-1}$, $564 \mathrm{~K} \mathrm{~km} \mathrm{~s}^{-1}, 846 \mathrm{~K} \mathrm{~km} \mathrm{~s}^{-1}, 1128 \mathrm{~K} \mathrm{~km} \mathrm{~s}^{-1}$ and $1410 \mathrm{~K} \mathrm{~km} \mathrm{~s}^{-1}$. The peak value of the ${ }^{12} \mathrm{CO}(3-2)$ map is $\int T_{\mathrm{mb}} \mathrm{d} v \approx 816 \mathrm{~K} \mathrm{~km} \mathrm{~s}^{-1}$. The peak value of the ${ }^{12} \mathrm{CO}(6-5)$ map is $\int T_{\mathrm{mb}} \mathrm{d} v \approx 1400 \mathrm{~K} \mathrm{~km} \mathrm{~s}^{-1}$. The maps are rotated by $51^{\circ}$ to match the major axis position angle of the $\mathrm{CO}$ emission in NGC 253 . The ${ }^{12} \mathrm{CO}(3-2)$ map has been shifted by $-5^{\prime \prime}$ in minor axis offset (see text in Sects. 3 and 4.1). The black box in the upper figure represents the size of the lower figure.

Nobeyama Interferometer (NRO), one near the center and two on each side at around $3^{\prime \prime}$ south-west and $8^{\prime \prime}$ north-east respectively (Paglione et al. 2004). The offset peak in the ${ }^{12} \mathrm{CO}(3-2)$ map may be associated with the north-east secondary peak. Tables 2 and 3 also report relevant data found in the literature. For some spectra, a Gaussian may not represent the true line profile but given the weakness of the signal, fitting anything more complicated is not warranted.

To be able to compare intensities of the different $\mathrm{CO}$ lines, we have also computed the line intensities ( $A$ and $I$ ) and fluxes $(F)$ for a common beam size of $21.9^{\prime \prime}$, which is the 
Table 2. Results for Henize 2-10.

\begin{tabular}{|c|c|c|c|c|c|c|}
\hline Henize 2-10 & & & & & & \\
\hline Transition & $\begin{array}{l}\text { Freq. } \\
(\mathrm{GHz})\end{array}$ & $\begin{array}{c}\text { Beam size } \\
\left({ }^{\prime \prime}\right)\end{array}$ & $\begin{array}{c}\int\left(T_{\mathrm{mb}} \mathrm{d} v\right) \\
\left(\mathrm{K} \mathrm{km} \mathrm{s}^{-1}\right)\end{array}$ & $\begin{array}{c}\text { Intensity } \\
\left(\mathrm{W} \mathrm{m}^{-2} \mathrm{sr}^{-1}\right)\end{array}$ & $\begin{array}{c}\text { Flux } \\
\left(\mathrm{W} \mathrm{m}^{-2}\right)\end{array}$ & References $^{a}$ \\
\hline \multirow[t]{2}{*}{$\mathrm{CI}\left({ }^{3} \mathrm{P}_{1}-{ }^{3} \mathrm{P}_{0}\right)$} & 492.162 & 14.55 & $4.2 \pm 0.8$ & $5.1 \times 10^{-10}$ & $3.0 \times 10^{-18}$ & 4 and $5^{b}$ \\
\hline & & 21.90 & $2.5 \pm 0.5$ & $3.0 \times 10^{-10}$ & $3.8 \times 10^{-18}$ & 4 and $5^{b}$ \\
\hline \multirow[t]{4}{*}{${ }^{12} \mathrm{CO}(1-0)$} & 115.271 & 40.00 & $10.0 \pm 0.8$ & $1.6 \times 10^{-11}$ & $6.7 \times 10^{-19}$ & 1 \\
\hline & & 21.90 & $27.3 \pm 2.2$ & $4.3 \times 10^{-11}$ & $5.5 \times 10^{-19}$ & 1 \\
\hline & & 55.00 & $4.9 \pm 0.2$ & $7.6 \times 10^{-12}$ & $6.1 \times 10^{-19}$ & 2 \\
\hline & & 21.90 & $23.9 \pm 1.0$ & $3.7 \times 10^{-11}$ & $4.8 \times 10^{-19}$ & $2 *$ \\
\hline \multirow[t]{3}{*}{${ }^{12} \mathrm{CO}(2-1)$} & 230.538 & 21.00 & $17.3 \pm 1.4$ & $2.2 \times 10^{-10}$ & $2.5 \times 10^{-18}$ & $1^{*}$ \\
\hline & & 27.00 & $6.8 \pm 0.8$ & $8.5 \times 10^{-11}$ & $1.7 \times 10^{-18}$ & 2 \\
\hline & & 21.90 & $9.4 \pm 1.1$ & $1.2 \times 10^{-10}$ & $1.5 \times 10^{-18}$ & 2 \\
\hline \multirow[t]{3}{*}{${ }^{12} \mathrm{CO}(3-2)$} & 345.796 & 21.90 & $11.5 \pm 2.3$ & $4.9 \times 10^{-10}$ & $6.2 \times 10^{-18}$ & $5^{*}$ \\
\hline & & 21.00 & $23.2 \pm 2.1$ & $9.8 \times 10^{-10}$ & $1.2 \times 10^{-17}$ & 1 \\
\hline & & 22.00 & $16.6 \pm 0.6$ & $7.0 \times 10^{-10}$ & $9.0 \times 10^{-18}$ & 3 \\
\hline \multirow[t]{2}{*}{${ }^{12} \mathrm{CO}(4-3)$} & 461.041 & 14.55 & $18.6 \pm 4.2$ & $1.9 \times 10^{-9}$ & $1.1 \times 10^{-17}$ & 5 \\
\hline & & 21.90 & $10.9 \pm 2.4$ & $1.1 \times 10^{-9}$ & $1.4 \times 10^{-17}$ & $5^{*}$ \\
\hline \multirow[t]{2}{*}{${ }^{12} \mathrm{CO}(6-5)$} & 691.473 & 10.60 & $15.7 \pm 3.1$ & $5.3 \times 10^{-9}$ & $1.6 \times 10^{-17}$ & 5 \\
\hline & & 21.90 & $6.8 \pm 1.3$ & $2.3 \times 10^{-9}$ & $2.9 \times 10^{-17}$ & $5^{*}$ \\
\hline \multirow[t]{2}{*}{${ }^{12} \mathrm{CO}(7-6)$} & 806.652 & 8.95 & $15.2 \pm 3.0$ & $8.2 \times 10^{-9}$ & $1.7 \times 10^{-17}$ & 5 \\
\hline & & 21.90 & $5.8 \pm 1.2$ & $3.1 \times 10^{-9}$ & $4.0 \times 10^{-17}$ & $5^{*}$ \\
\hline \multirow[t]{4}{*}{${ }^{13} \mathrm{CO}(1-0)$} & 110.201 & 40.00 & $<0.5$ & $<6.8 \times 10^{-13}$ & $<2.9 \times 10^{-20}$ & 1 \\
\hline & & 21.90 & $<1.4$ & $<1.8 \times 10^{-12}$ & $<2.4 \times 10^{-20}$ & 1 \\
\hline & & 57.00 & $0.3 \pm 0.1$ & $3.9 \times 10^{-13}$ & $3.4 \times 10^{-20}$ & 2 \\
\hline & & 21.90 & $1.6 \pm 0.5$ & $2.2 \times 10^{-12}$ & $2.8 \times 10^{-20}$ & $2 *$ \\
\hline${ }^{13} \mathrm{CO}(2-1)$ & 220.399 & 21.00 & $0.9 \pm 0.2$ & $9.9 \times 10^{-12}$ & $1.2 \times 10^{-19}$ & $1 *$ \\
\hline \multirow[t]{3}{*}{${ }^{13} \mathrm{CO}(3-2)$} & 330.588 & 14.00 & $2.3 \pm 0.6$ & $8.5 \times 10^{-11}$ & $4.4 \times 10^{-19}$ & 1 \\
\hline & & 21.90 & $1.3 \pm 0.3$ & $4.8 \times 10^{-11}$ & $6.1 \times 10^{-19}$ & $1 *$ \\
\hline & & 21.90 & $1.8 \pm 0.5$ & $6.7 \times 10^{-11}$ & $8.5 \times 10^{-19}$ & 5 \\
\hline
\end{tabular}

${ }^{a}$ References: 1: Baas et al. (1994); 2: Kobulnicky et al. (1995); 3: Meier et al. (2001); 4: Gerin \& Phillips (2000); 5: this work; *: used for models.

${ }^{b}$ We analysed again spectra from Gerin \& Phillips (2000).

size of the ${ }^{12} \mathrm{CO}(3-2)$ beam at the CSO. They are listed in Tables 2 and 3 and identified with an asterisk in Col. 7 (named References). To do so, we have used the following assumptions:

- Henize 2-10: we modelled the source with an axisymmetric Gaussian distribution, with a full width half maximum (FWHM) of 13", as found by Meier et al. (2001).

- NGC 253: we made use of the ${ }^{12} \mathrm{CO}(6-5)$ map to determine the source size at $690 \mathrm{GHz}$ and model the emission at higher frequencies. We modelled the nucleus as an elliptical source with Gaussian intensity profiles. The ${ }^{12} \mathrm{CO}(6-5)$ map can be well fitted with such a model, with half maximum width of $23^{\prime \prime}$ along the major axis and 11" along the minor axis, which is very similar to the size of the CS $(J=2-1)$ emission in the nucleus (Peng et al. 1996).
The source appears to be resolved along the major axis but unresolved along the minor axis. We used the same source model for ${ }^{12} \mathrm{CO}(7-6)$ and $\mathrm{CI}\left({ }^{3} \mathrm{P}_{2}-{ }^{3} \mathrm{P}_{1}\right)$, as it fits our restricted data set.

- $\boldsymbol{I}$ is derived using the formula:

$I=\frac{2 \times k_{\mathrm{b}} \times v^{3}}{c^{3}} \times A$

$I\left(\mathrm{~W} \mathrm{~m}^{-2} \mathrm{sr}^{-1}\right)=1.02 \times 10^{-18} \times\left(\frac{v}{\mathrm{GHz}}\right)^{3} \times\left(\frac{A}{\mathrm{~K} \mathrm{~km} \mathrm{~s}^{-1}}\right)$

where $c$ is the speed of light, $v$ is the line frequency in $\mathrm{GHz}$ and $A$ is the line area in $\mathrm{K} \mathrm{km} \mathrm{s}^{-1}$.

To derive the flux, $F$ in $\mathrm{W} \mathrm{m}^{-2}$, we multiply $I$ in $\mathrm{W} \mathrm{m}^{-2} \mathrm{sr}^{-1}$ by the beam solid angle $\Omega(B)$ in $s r$ defined as:

$\Omega(B)=1.133 \times B^{2} \times \frac{1}{206265^{2}} \mathrm{sr}$ 
Table 3. Results for the nucleus of NGC 253.

\begin{tabular}{|c|c|c|c|c|c|c|}
\hline NGC 253 & & & & & & \\
\hline Transition & $\begin{array}{c}\text { Freq } \\
(\mathrm{GHz})\end{array}$ & $\begin{array}{c}\text { Beam size } \\
\left({ }^{\prime \prime}\right)\end{array}$ & $\begin{array}{c}\int\left(T_{\mathrm{mb}} \mathrm{d} v\right) \\
\left(\mathrm{K} \mathrm{km} \mathrm{s}^{-1}\right)\end{array}$ & $\begin{array}{c}\text { Intensity } \\
\left(\mathrm{W} \mathrm{m}^{-2} \mathrm{sr}^{-1}\right)\end{array}$ & $\begin{array}{c}\text { Flux } \\
\left(\mathrm{W} \mathrm{m}^{-2}\right)\end{array}$ & References $^{a}$ \\
\hline \multirow{5}{*}{$\mathrm{CI}\left({ }^{3} \mathrm{P}_{1}-{ }^{3} \mathrm{P}_{0}\right)$} & 492.162 & 10.20 & $575.0 \pm 115.0^{b}$ & $7.0 \times 10^{-8}$ & $1.9 \times 10^{-16}$ & 5 \\
\hline & & 21.90 & $204.0 \pm 41.0$ & $2.5 \times 10^{-8}$ & $3.2 \times 10^{-16}$ & 5 \\
\hline & & 43.00 & $98.0 \pm 19.6^{b}$ & $1.2 \times 10^{-8}$ & $5.9 \times 10^{-16}$ & 5 \\
\hline & & 22.00 & $290.0 \pm 45$ & $3.5 \times 10^{-8}$ & $4.6 \times 10^{-16}$ & $10^{*}$ \\
\hline & & 23.00 & $320.0 \pm 64.0^{b}$ & $3.9 \times 10^{-8}$ & $5.5 \times 10^{-16}$ & 4 \\
\hline \multirow[t]{2}{*}{$\mathrm{CI}\left({ }^{3} \mathrm{P}_{2}-{ }^{3} \mathrm{P}_{1}\right)$} & 809.902 & 8.95 & $188.5 \pm 37.7$ & $1.0 \times 10^{-7}$ & $2.2 \times 10^{-16}$ & 11 \\
\hline & & 21.9 & $58.1 \pm 11.6$ & $3.2 \times 10^{-8}$ & $4.0 \times 10^{-16}$ & $11^{*}$ \\
\hline \multirow[t]{2}{*}{${ }^{12} \mathrm{CO}(1-0)$} & 115.271 & 43.00 & $343.0 \pm 68.6^{b}$ & $5.4 \times 10^{-10}$ & $2.6 \times 10^{-17}$ & 2 \\
\hline & & 23.00 & $920.0 \pm 82.8$ & $1.4 \times 10^{-9}$ & $2.0 \times 10^{-17}$ & $6^{*}$ \\
\hline \multirow[t]{2}{*}{${ }^{12} \mathrm{CO}(2-1)$} & 230.538 & 23.00 & $1062.0 \pm 116.8$ & $1.3 \times 10^{-8}$ & $1.9 \times 10^{-16}$ & $7 *$ \\
\hline & & 21.00 & $926.0 \pm 185.2^{b}$ & $1.2 \times 10^{-8}$ & $1.4 \times 10^{-16}$ & 2 \\
\hline \multirow[t]{6}{*}{${ }^{12} \mathrm{CO}(3-2)$} & 345.796 & 21.90 & $815.6 \pm 163.1$ & $3.5 \times 10^{-8}$ & $4.4 \times 10^{-16}$ & $11^{*}$ \\
\hline & & 23.00 & $998.0 \pm 139.7$ & $4.2 \times 10^{-8}$ & $5.9 \times 10^{-16}$ & 7 \\
\hline & & 23.00 & $1194.0 \pm 238.8^{b}$ & $5.0 \times 10^{-8}$ & $7.1 \times 10^{-16}$ & 2 \\
\hline & & 14.00 & $1200.0 \pm 240.0^{b}$ & $5.1 \times 10^{-8}$ & $2.6 \times 10^{-16}$ & 5 \\
\hline & & 21.90 & $618.9 \pm 123.8$ & $2.6 \times 10^{-8}$ & $3.3 \times 10^{-16}$ & 5 \\
\hline & & 22.00 & $680.0 \pm 60.0$ & $2.9 \times 10^{-8}$ & $3.7 \times 10^{-16}$ & 8 \\
\hline \multirow[t]{5}{*}{${ }^{12} \mathrm{CO}(4-3)$} & 461.041 & 15.00 & $507.0 \pm 101.4^{b}$ & $5.1 \times 10^{-8}$ & $3.0 \times 10^{-16}$ & 3 \\
\hline & & 21.90 & $285.3 \pm 57.1$ & $2.9 \times 10^{-8}$ & $3.7 \times 10^{-16}$ & 3 \\
\hline & & 22.00 & $1019.0 \pm 120$ & $1.0 \times 10^{-7}$ & $1.3 \times 10^{-15}$ & $9 *$ \\
\hline & & 10.40 & $2160.0 \pm 432.0^{b}$ & $2.1 \times 10^{-7}$ & $6.2 \times 10^{-16}$ & 5 \\
\hline & & 21.90 & $786.2 \pm 157.2$ & $7.9 \times 10^{-7}$ & $1.0 \times 10^{-15}$ & 5 \\
\hline \multirow[t]{3}{*}{${ }^{12} \mathrm{CO}(6-5)$} & 691.473 & 10.60 & $1394 \pm 278.8$ & $4.7 \times 10^{-7}$ & $1.4 \times 10^{-15}$ & 11 \\
\hline & & 21.90 & $518.3 \pm 104.3$ & $1.8 \times 10^{-7}$ & $2.2 \times 10^{-15}$ & $11^{*}$ \\
\hline & & $8 / 30$ & $861 \pm 258.3$ & $2.9 \times 10^{-7}$ & $5.0 \times 10^{-15}$ & 1 \\
\hline \multirow[t]{3}{*}{${ }^{12} \mathrm{CO}(7-6)$} & 806.652 & 8.95 & $810.2 \pm 162.0$ & $4.3 \times 10^{-7}$ & $9.3 \times 10^{-16}$ & 11 \\
\hline & & 21.90 & $249.9 \pm 50.3$ & $1.3 \times 10^{-7}$ & $1.7 \times 10^{-15}$ & $11^{*}$ \\
\hline & & $11.5 / 60$ & $1370 \pm 411$ & $7.3 \times 10^{-7}$ & $2.6 \times 10^{-15}$ & 10 \\
\hline${ }^{13} \mathrm{CO}(1-0)$ & 110.201 & 23.00 & $80 \pm 8.0$ & $1.1 \times 10^{-10}$ & $1.5 \times 10^{-18}$ & $7 *$ \\
\hline \multirow[t]{2}{*}{${ }^{13} \mathrm{CO}(2-1)$} & 220.399 & 23.00 & $82 \pm 9.8$ & $9.0 \times 10^{-10}$ & $1.3 \times 10^{-17}$ & $7 *$ \\
\hline & & 21.00 & $104 \pm 20.8^{b}$ & $1.1 \times 10^{-9}$ & $1.3 \times 10^{-17}$ & 2 \\
\hline \multirow[t]{2}{*}{${ }^{13} \mathrm{CO}(3-2)$} & 330.588 & 23.00 & $90 \pm 12.6$ & $3.3 \times 10^{-9}$ & $4.7 \times 10^{-17}$ & $7 *$ \\
\hline & & 23.00 & $210 \pm 42.0^{b}$ & $7.8 \times 10^{-9}$ & $1.1 \times 10^{-16}$ & 2 \\
\hline
\end{tabular}

${ }^{a}$ References: 1: Harris et al. (1991); 2: Wall et al. (1991); 3: Guesten et al. (1993); 4: Harrison et al. (1995); 5: Israel et al. (1995); 6: Mauersberger et al. (1996); 7: Harrison et al. (1999); 8: Dumke et al. (2001); 9: Israel \& Baas (2002); 10: Bradford et al. (2003); 11: this work; *: used for models.

${ }^{b}$ The error is not given in the referenced paper. It has been estimated to be $20 \%$.

where $B$ is the half power beam width (HPBW) in arcsec. The estimated error for our data in Tables 2 and 3 is about $20 \%$.

\section{2. $C$ and $C O$ cooling}

Our observations are designed to provide essential information on the cooling and consequently on the thermal balance of the interstellar medium in Henize 2-10 and NGC 253. We shall also deduce which CO line(s) contribute the most to the total observed CO cooling and estimate the total observed cooling of $\mathrm{C}$ and $\mathrm{CO}$ by summing intensities in $\mathrm{W} \mathrm{m}^{-2} \mathrm{sr}^{-1}$ of all transitions listed in Tables 2 and 3 with asterisks (both literature data and our dataset). We have computed the observed $\mathrm{C}$ and $\mathrm{CO}$ cooling in the galaxy nuclei for a beam size of $21.9^{\prime \prime}$, this corresponds to linear scales of $640 \mathrm{pc}$ and $270 \mathrm{pc}$ for Henize 2-10 and NGC 253 respectively.

For Henize 2-10 and NGC 253 we measured a total observed $\mathrm{CO}$ cooling rate of $7.2 \times 10^{-9} \mathrm{~W} \mathrm{~m}^{-2} \mathrm{sr}^{-1}$ and $4.6 \times 10^{-7} \mathrm{~W} \mathrm{~m}^{-2} \mathrm{sr}^{-1}$ respectively. For NGC 253 
the lines contributing the most to the observed $\mathrm{CO}$ cooling are ${ }^{12} \mathrm{CO}(6-5)(39.2 \%$ of the total intensity) followed by ${ }^{12} \mathrm{CO}(7-6)(28.3 \%)$. For Henize $2-10$, it is ${ }^{12} \mathrm{CO}(7-6)(43.1 \%)$ followed by ${ }^{12} \mathrm{CO}(6-5)(31.9 \%)$.

The observed total cooling rates of neutral carbon $\mathrm{C}$ for Henize 2-10 and NGC 253 are respectively $3.0 \times$ $10^{-10} \mathrm{~W} \mathrm{~m}^{-2} \mathrm{sr}^{-1}\left(\mathrm{CI}\left({ }^{3} \mathrm{P}_{1}-{ }^{3} \mathrm{P}_{0}\right)\right.$ transition only) and $6.7 \times$ $10^{-8} \mathrm{~W} \mathrm{~m}^{-2} \mathrm{sr}^{-1}$ (for NGC $253, \mathrm{CI}\left({ }^{3} \mathrm{P}_{2}-{ }^{3} \mathrm{P}_{1}\right.$ ) represents $52.2 \%$ of the total).

These results show that ${ }^{12} \mathrm{CO}(6-5)$ and ${ }^{12} \mathrm{CO}(7-6)$ are contributing the most to the total observed $\mathrm{CO}$ cooling, with very similar percentages for both galaxies. It is natural to wonder whether ${ }^{12} \mathrm{CO}(5-4)$ and ${ }^{12} \mathrm{CO}(8-7)$ are strong also. This will be investigated in Sects. 5.2 and 5.3.

In starburst nuclei, $\mathrm{CO}$ cooling is larger than $\mathrm{C}$ cooling by a factor of $>10$, which explains why it is easier to detect $\mathrm{CO}$ than $\mathrm{C}$ in distant galaxies. Similar results have been obtained on $\mathrm{J} 1148+5251(z=6.42)$ and on PSS2322+1944 $(z=4.12)$ (Bertoldi et al. 2003; Walter et al. 2003; Cox et al. 2002). Barvainis et al (1997) detected $\mathrm{Cl}\left({ }^{3} \mathrm{P}_{1}-{ }^{3} \mathrm{P}_{0}\right)(3.6 \pm$ $0.4 \mathrm{Jy} \mathrm{km} \mathrm{s}^{-1}$ ) in the Cloverleaf quasar at redshift $z=2.5$. In the same quasar, Weiß et al. (2003) detected $\mathrm{CI}\left({ }^{3} \mathrm{P}_{2}-{ }^{3} \mathrm{P}_{1}\right)$ $\left(5.2 \pm 0.3 \mathrm{Jy} \mathrm{km} \mathrm{s}^{-1}\right)$ and ${ }^{12} \mathrm{CO}(3-2)\left(13.2 \pm 0.2 \mathrm{Jy} \mathrm{km} \mathrm{s}^{-1}\right)$. In distant objects, $\mathrm{C}$ cooling also seems to be weaker than CO cooling.

\section{CO models}

In this section, we use the measured $\mathrm{CO}$ line ratios and intensities ( $I$ and $A$ ) to determine the physical conditions of molecular gas, namely the kinetic temperature, the gas density, the CO column density and the FUV flux: $\chi$. In the first section, we start with an LTE analysis. In Sect. 5.2, we use an LVG radiative transfer model and in Sect. 5.3, we discuss the use of a PDR model. In the last section, we discuss the similarities and differences between the two galaxies, the nucleus of the Milky Way and the distant QSO "the Cloverleaf". The latter has been chosen as being representative of distant, actively star forming galaxies.

\subsection{LTE analysis}

In the local thermodynamical equilibrium (LTE) approximation, we assume that $\mathrm{CO}$ is thermalized, hence the relative populations of its energy levels are functions of the kinetic temperature (assumed uniform) only. We discuss two limiting cases, applied to ${ }^{12} \mathrm{CO}$ and ${ }^{13} \mathrm{CO}$ :

$\diamond$ the lines are optically thick (optical depth: $\tau \gg 1$ ); the line intensity ratio (e.g. $\frac{{ }^{12} \mathrm{CO}(3-2)}{{ }^{12} \mathrm{CO}(2-1)}$ can be written:

$R_{32 / 21}^{\text {thick }}=\frac{F F \times T^{12} \mathrm{CO}(3-2)}{F F \times T^{12} \mathrm{CO}(2-1)}=\frac{J_{v 32}\left(T_{\mathrm{k}}\right)-J_{v 32}\left(T_{\mathrm{bg}}\right)}{J_{v 21}\left(T_{\mathrm{k}}\right)-J_{v 21}\left(T_{\mathrm{bg}}\right)}$

with $J_{v}\left(T_{\mathrm{k}}\right)$ defined as in Eq. (2) in Sect. 1. In LTE, $R_{32 / 21}^{\text {thick }} \approx$ 1 and $R_{76 / 65}^{\text {thick }} \approx 1$ for $T_{\mathrm{k}}>30 \mathrm{~K}$, while $R_{65 / 32}^{\text {thick }} \approx 1$ and $R_{76 / 32}^{\text {thick }} \approx 1$ for $T_{\mathrm{k}}>100 \mathrm{~K}$. Plots of the line intensity ratios $\left(R^{\text {thick}}\right)$ as a function of the kinetic temperature $T_{\mathrm{k}}$ for the

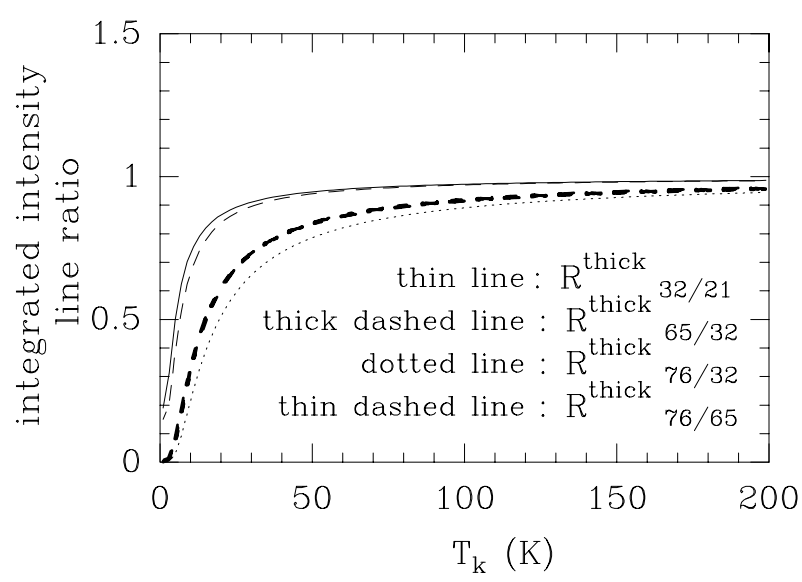

Fig. 4. Integrated intensity lines ratios, $R^{\text {thick }}$, (defined as in Eq. (6)), vs. kinetic temperature in K. We assume LTE, and optically thick lines (optical depth, $\tau \gg 1$ ).

optically thick case in the LTE approximation are given in Fig. 4. From this figure, we conclude that the line ratios combining high- $J\left({ }^{12} \mathrm{CO}(6-5)\right.$ or $\left.{ }^{12} \mathrm{CO}(7-6)\right)$ with low- $J$ $\left({ }^{12} \mathrm{CO}(3-2)\right)$ lines are the most useful for constraining the kinetic temperature.

$\diamond$ One line is optically thick and the other is optically thin; this is likely when comparing line intensities for different isotopologues of $\mathrm{CO}$ in the same $J$ transition (e.g. $\left.{ }^{12} \mathrm{CO}(3-2) /{ }^{13} \mathrm{CO}(3-2)\right)$. Assuming equal excitation temperatures for both species, optically thin ${ }^{13} \mathrm{CO}$ lines and optically thick ${ }^{12} \mathrm{CO}$ lines, we get:

$$
\begin{aligned}
R_{12 / 13} & =\frac{F F \times T^{12} \mathrm{CO}}{F F \times T^{13} \mathrm{CO}} \approx \frac{A\left({ }^{12} \mathrm{CO}\right)}{A\left({ }^{13} \mathrm{CO}\right)} \approx \frac{1}{\tau\left({ }^{13} \mathrm{CO}\right)} \\
& \approx \frac{X_{\text {galaxy }}}{\tau\left({ }^{12} \mathrm{CO}\right)}
\end{aligned}
$$

where $X_{\text {galaxy }}$ is the ${ }^{12} \mathrm{CO} /{ }^{13} \mathrm{CO}$ abundance ratio. Previous work, using LVG models, found, for Henize 2-10, $X \simeq$ 30 (Baas et al. 1994) and for NGC 253, $X \simeq 40$ (Henkel \& Mauersberger 1993; Henkel et al. 1993; Israel $\&$ Baas 2002). From the literature data, summarized in Tables 2, and 3, we deduced $\tau\left({ }^{12} \mathrm{CO}(1-0)\right) \simeq 2.0$ and $\tau\left({ }^{12} \mathrm{CO}(2-1)\right) \simeq 1.6$ for Henize 2-10. For NGC 253, we obtained $\tau\left({ }^{12} \mathrm{CO}(1-0)\right) \simeq 3.5$ and $\tau\left({ }^{12} \mathrm{CO}(2-1)\right) \simeq 3.1$ $\left(\tau\right.$ increase for larger $X_{\text {galaxy }}$ : assuming $X_{\mathrm{NGC}} 253 \simeq 50$ as in Bradford et al. (2003) $\tau\left({ }^{12} \mathrm{CO}(1-0)\right) \simeq 4.3$ and $\tau\left({ }^{12} \mathrm{CO}(2-1)\right) \simeq 3.9$.

Unfortunately, the opacity of the ${ }^{12} \mathrm{CO}(3-2)$ line for both galaxies is not accurately determined due to systematic differences between data sets (Baas et al. 1994; Harrison et al. 1999, and this work). For Henize $2-10, \tau\left({ }^{12} \mathrm{CO}(3-2)\right)$ ranges between 1.7 and 3.4, and for NGC 253, it ranges between 3.0 and 5.8 for the abundance ratio given above $\left(X_{\mathrm{NGC} 253}=40\right)$. We note that the largest error is for the ${ }^{13} \mathrm{CO}$ integrated intensity which is the most difficult line to observe: for NGC 253 , A $\left({ }^{13} \mathrm{CO}(3-2)\right)$ varies between $90 \mathrm{~K} \mathrm{~km} \mathrm{~s}^{-1}$ (Harrison et al. 1999) and $210 \mathrm{~K} \mathrm{~km} \mathrm{~s}^{-1}$ (Wall et al. 1991) while $\mathrm{A}\left({ }^{12} \mathrm{CO}(3-2)\right)$ varies between 
$619 \mathrm{~K} \mathrm{~km} \mathrm{~s}^{-1}$ (Israel et al. 1995) and $1194 \mathrm{~K} \mathrm{~km} \mathrm{~s}^{-1}$ (Wall et al. 1991) (at the same resolution).

Because the observed ratio is $R_{65 / 32}^{\text {thick }} \approx 0.6$ for both Henize 2-10 and NGC 253 (see Table 4), we conclude, from the LTE analysis, that the ${ }^{12} \mathrm{CO}(3-2)$ line opacities are moderate $(1<\tau<10)$ and the gas is warm $\left(T_{\mathrm{k}}>30 \mathrm{~K}\right)$.

To go further and constrain the gas density, we now use LVG models.

\subsection{LVG model}

The detection of the high-excitation CO lines directly indicates the presence of large amounts of warm and dense gas in these galaxies. The CO excitation is modelled in terms of standard, one-component, spherical LVG radiative transfer model with uniform kinetic temperature and density (Goldreich \& Kwan 1974; De Jong et al. 1975). Though the assumption of uniform physical conditions is crude, these models represent a useful step in predicting intensities of the submillimeter CO lines, since, in any case, we lack information concerning the gas distribution in the galaxy, particularly its small scale structure.

There are four main variables in LVG models: the CO column density divided by the line width: $N\left({ }^{12} \mathrm{CO}\right) / \Delta v$, the molecular hydrogen density: $n\left(\mathrm{H}_{2}\right)$, the kinetic temperature: $T_{\mathrm{k}}$ and the $\frac{{ }^{12} \mathrm{CO}}{{ }^{13} \mathrm{CO}}$ abundance ratio: $X_{\text {galaxy }}$. The linewidth, $\Delta v$, is constrained by the fit of the ${ }^{12} \mathrm{CO}(3-2)$ spectra $(F W H M)$. We have used $\Delta v=60 \mathrm{~km} \mathrm{~s}^{-1}$ for Henize 2-10 and $\Delta v=190 \mathrm{~km} \mathrm{~s}^{-1}$ for NGC 253 (see Fig. 2). The $\frac{{ }^{12} \mathrm{CO}}{{ }^{13} \mathrm{CO}}$ abundance ratio varies between 30 and 40 for Henize 2-10 $\left(X_{\text {Henize 2-10 }}\right)$ and between 30 and 50 for NGC $253\left(X_{\mathrm{NGC} 253}\right)$.

To make quantitative estimates of the physical parameters of the molecular gas in nuclei, we have compiled information about line emission for all $\mathrm{CO}$ rotational transitions observed so far and scaled them to a common beam size of 21.9" (see Tables 2 and 3 and Sect. 4.1).

Model solutions for the physical parameters, for both sources, are presented in Table 5. Because acceptable fits can be obtained over a large domain of parameter space, we have chosen to present two models which bracket the range of possible solutions for the kinetic temperature. The first one has a "low kinetic temperature" $\left(T_{\mathrm{k}}<80 \mathrm{~K}\right)$, shown with crosses in Figs. 5 and 6 and the second one has a "high $T_{\mathrm{k}}$ " $\left(T_{\mathrm{k}}>100 \mathrm{~K}\right)$, shown with triangles in Figs. 5 and 6 (see Table 4). The gas density is not well constrained but must be at least $10^{4} \mathrm{~cm}^{-3}$. The best fit ${ }^{12} \mathrm{CO} /{ }^{13} \mathrm{CO}$ value is 30 for Henize $2-10$ and 40 for NGC 253 , both compatible with previous analyses. We varied $N\left({ }^{12} \mathrm{CO}\right)$ from $1.0 \times 10^{16} \mathrm{~cm}^{-2}$ to $1.0 \times 10^{20} \mathrm{~cm}^{-2}$ for Henize $2-10$ and $3.5 \times 10^{17} \mathrm{~cm}^{-2}$ to $3.5 \times 10^{19} \mathrm{~cm}^{-2}$ for NGC 253 . CO column densities are mainly constrained by the ${ }^{13} \mathrm{CO}$ data (see Tables 2 and 3). Acceptable fits are found: $N\left({ }^{12} \mathrm{CO}\right)=3.5 \pm 1 \times 10^{18} \mathrm{~cm}^{-2}$ for Henize 2-10 and for $N\left({ }^{12} \mathrm{CO}\right)=1.5 \pm 0.5 \times 10^{19} \mathrm{~cm}^{-2}$ for NGC 253. We determined the "best" fit using a least square fit method for the line area ratios listed in Table 4. As we wanted to derive the properties of warm gas, we have given more weight to line ratios which include high- $J$ CO lines, e.g.

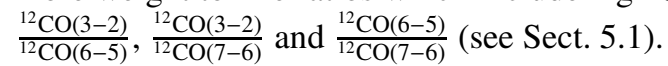

Table 4. Observed and predicted ("low $T_{\mathrm{k}}$ " and "high $T_{\mathrm{k}}$ " LVG models) line area ratios for Henize 2-10 and NGC 253.

\begin{tabular}{|c|c|c|}
\hline $\begin{array}{c}A \\
\left(\mathrm{~K} \mathrm{~km} \mathrm{~s}^{-1}\right)\end{array}$ & $\begin{array}{c}\text { NGC } 253 \\
\text { observations }\end{array}$ & $\begin{array}{l}\text { Henize } 2-10 \\
\text { observations }\end{array}$ \\
\hline$\frac{12 \mathrm{CO}(3-2)}{{ }^{12} \mathrm{CO}(6-5)}$ & $1.6 \pm 0.6^{*}$ & $1.7 \pm 0.7^{*}$ \\
\hline$\frac{{ }^{12} \mathrm{CO}(3-2)}{{ }^{12} \mathrm{CO}(7-6)}$ & $3.3 \pm 1.3^{*}$ & $2.0 \pm 0.8^{*}$ \\
\hline$\frac{{ }^{12} \mathrm{CO}(6-5)}{{ }^{12} \mathrm{CO}(7-6)}$ & $2.1 \pm 0.8^{*}$ & $1.2 \pm 0.5^{*}$ \\
\hline$\frac{{ }^{12} \mathrm{CO}(1-0)}{{ }^{13} \mathrm{CO}(1-0)}$ & $11.5 \pm 2.2^{*}$ & $14.9 \pm 5.8^{*}$ \\
\hline$\frac{{ }^{12} \mathrm{CO}(2-1)}{{ }^{13} \mathrm{CO}(2-1)}$ & $13.0 \pm 3.0^{*}$ & $19.2 \pm 5.8^{*}$ \\
\hline \multirow[t]{3}{*}{$\frac{{ }^{12} \mathrm{CO}(3-2)}{{ }^{13} \mathrm{CO}(3-2)}$} & $9.1 \pm 3.1^{*}$ & $8.9 \pm 3.8^{*}$ \\
\hline & "Low $T_{\mathrm{k}}$ " model & "Low $T_{\mathrm{k}}$ " model \\
\hline & $T_{\mathrm{k}}=70 \mathrm{~K}$ & $T_{\mathrm{k}}=50 \mathrm{~K}$ \\
\hline$\frac{{ }^{12} \mathrm{CO}(3-2)}{{ }^{12} \mathrm{CO}(6-5)}$ & 1.6 & 2.0 \\
\hline$\frac{{ }^{12} \mathrm{CO}(3-2)}{{ }^{12} \mathrm{CO}(7-6)}$ & 2.5 & 3.8 \\
\hline$\frac{{ }^{12} \mathrm{CO}(6-5)}{{ }^{12} \mathrm{CO}(7-6)}$ & 1.5 & 1.9 \\
\hline$\frac{{ }^{12} \mathrm{CO}(1-0)}{{ }^{13} \mathrm{CO}(1-0)}$ & 25.5 & 19.1 \\
\hline$\frac{{ }^{12} \mathrm{CO}(2-1)}{{ }^{13} \mathrm{CO}(2-1)}$ & 13.4 & 9.9 \\
\hline \multirow[t]{3}{*}{$\frac{{ }^{12} \mathrm{CO}(3-2)}{{ }^{13} \mathrm{CO}(3-2)}$} & 10.1 & 8.1 \\
\hline & "High $T_{\mathrm{k}}$ " model & "High $T_{\mathrm{k}}$ " model \\
\hline & $T_{\mathrm{k}}=150 \mathrm{~K}$ & $T_{\mathrm{k}}=100 \mathrm{~K}$ \\
\hline$\frac{12 \mathrm{CO}(3-2)}{\frac{12}{\mathrm{CO}(6-5)}}$ & 1.4 & 1.6 \\
\hline$\frac{{ }^{12} \mathrm{CO}(3-2)}{{ }^{12} \mathrm{CO}(7-6)}$ & 1.8 & 2.5 \\
\hline$\frac{12 \mathrm{CO}(6-5)}{12 \mathrm{CO}(7-6)}$ & 1.3 & 1.5 \\
\hline$\frac{{ }^{12} \mathrm{CO}(1-0)}{{ }^{13} \mathrm{CO}(1-0)}$ & 15.1 & 23.9 \\
\hline$\frac{{ }^{12} \mathrm{CO}(2-1)}{{ }^{13} \mathrm{CO}(2-1)}$ & 20.7 & 14.8 \\
\hline$\frac{{ }^{12} \mathrm{CO}(3-2)}{{ }^{13} \mathrm{CO}(3-2)}$ & 14.8 & 9.9 \\
\hline
\end{tabular}

* Ratio derived from observations marked with asterisks in Tables 2 and 3 .

Predicted line integrated areas, $\mathrm{A}$ in $\mathrm{K} \mathrm{km} \mathrm{s}^{-1}$ and line intensities $I$ in $\mathrm{W} \mathrm{m}^{-2} \mathrm{sr}^{-1}$ for all CO transitions to $J=15-14$, are also shown in Figs. 5 and 6 . The predicted line area A is obtained by multiplying the antenna temperature, given by models, by the line width $\Delta v$ and by the surface filling factor, $F F$. For each observed CO line, we estimated the surface filling factor $(F F)$ of molecular clouds in the beam using the ratio $T_{\mathrm{mb}}$ (observations) $/ T_{\mathrm{mb}}$ (model). We made an average, weighted by the $\mathrm{S} / \mathrm{N}$, for the $F F$ pertaining to each observed transition. For Henize 2-10 and NGC 253, we obtained filling factors of $F F=8.8 \pm 3 \times 10^{-3}$ and $F F=8.7 \pm 3 \times 10^{-2}$ respectively. The line intensities, $I$, are derived from the model A values through Eqs. (3) and (4). We give in Table 4, line intensity ratios of $\mathrm{CO}$ transitions derived from observations and from models.

The low- $J$ CO transitions $\left({ }^{12} \mathrm{CO}(1-0)\right.$ and $\left.{ }^{12} \mathrm{CO}(2-1)\right)$ are not well fit by the adopted LVG models, because we have chosen to constrain the models with high- $J$ transitions of $\mathrm{CO}$ as we are interested in the warm gas properties. In order to study the properties of the low excitation molecular gas, we could have 

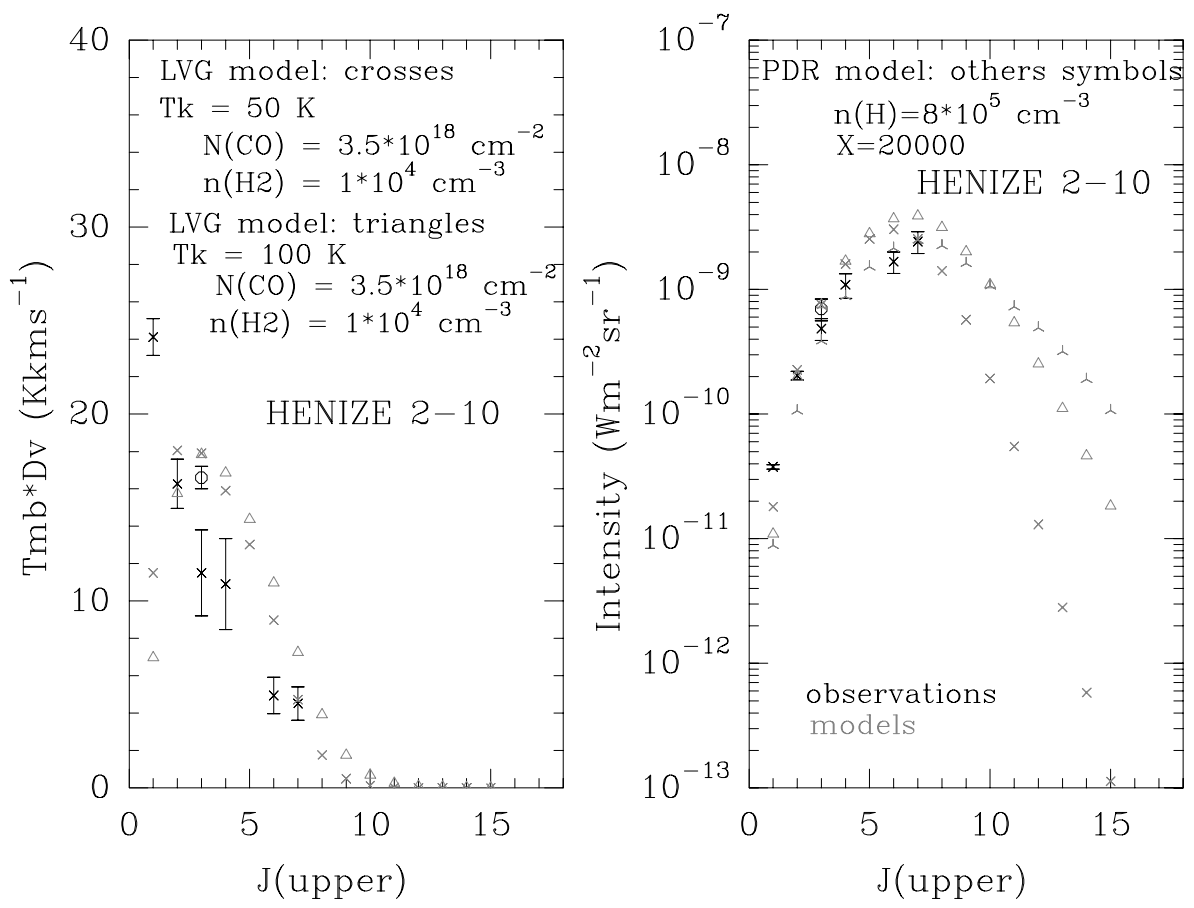

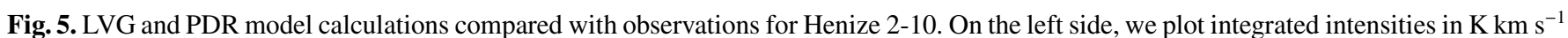
vs. $J_{\text {upper }}$. On the right side, we plot $I$ in $\mathrm{W} \mathrm{m}^{-2} \mathrm{sr}^{-1} \mathrm{vs.} J_{\text {upper. }}$. In both figures, grey crosses represent the "low temperature" LVG model while grey triangles represent the "high temperature" LVG model. The starred grey triangles represent results of the PDR model (see Sect. 5.3). Observations (with error bars) taken from literature and from our data set are shown in black. We have reported two observations for ${ }^{12} \mathrm{CO}(3-2)$ in Henize 2-10: the black cross is from our data and the black circle is from Meier et al. (2001). The last one seems to be agree better with the model predictions. As Henize 2-10 is a point source, a small pointing error can explain this difference.

Table 5. Parameters of the "best" LVG models.

\begin{tabular}{c|cc}
\hline \hline & Henize 2-10 & NGC 253 \\
\hline$n\left(\mathrm{H}_{2}\right)\left(\mathrm{cm}^{-3}\right)$ & $\gtrsim 1 \times 10^{4}$ & $\gtrsim 1 \times 10^{4}$ \\
$N\left({ }^{12} \mathrm{CO}\right)\left(\mathrm{cm}^{-2}\right)$ & $3.5 \pm 1 \times 10^{18}$ & $1.5 \pm 0.5 \times 10^{19}$ \\
$\frac{12 \mathrm{C}}{{ }^{13} \mathrm{C}}$ & 30 & 40 \\
$\Delta v^{*}\left(\mathrm{~km} \mathrm{~s}^{-1}\right)$ & 60 & 187 \\
$T_{\mathrm{k}}(\mathrm{K})$ & $50-100$ & $70-150$ \\
$F F$ & $8.8 \pm 3 \times 10^{-3}$ & $8.7 \pm 3 \times 10^{-2}$ \\
\hline
\end{tabular}

* Deduced from Gaussian fits to the ${ }^{12} \mathrm{CO}(3-2)$ line profiles.

introduced another gas component in the models as in Harrison et al. (1999) or Bradford et al. (2003) for NGC 253. Here, the LVG models predict too low an intensity for the ${ }^{12} \mathrm{CO}(1-0)$ and ${ }^{12} \mathrm{CO}(2-1)$ lines in this source, supporting the need for a low excitation gas component. For NGC 253, we obtained a similar kinetic temperature $T_{\mathrm{k}}$ as in Bradford et al. (2003), but a lower density $n\left(\mathrm{H}_{2}\right)$ and a higher $\mathrm{CO}$ column density $N\left({ }^{12} \mathrm{CO}\right)$. For NGC 253, Paglione et al. (2004) found good fits with LVG models with $N\left({ }^{12} \mathrm{CO}\right) / \Delta v$ between $4.0 \times 10^{16} \mathrm{~cm}^{-2} \mathrm{~km}^{-1} \mathrm{~s}$ and $1.3 \times 10^{18} \mathrm{~cm}^{-2} \mathrm{~km}^{-1} \mathrm{~s}$, in good agreement with our value $\left(N\left({ }^{12} \mathrm{CO}\right) / \Delta v=8.0 \times 10^{16} \mathrm{~cm}^{-2} \mathrm{~km}^{-1} \mathrm{~s}\right)$. Recent measurements of rotational lines of $\mathrm{H}_{2}$ (Rigopoulou et al. 2002) suggest that warm gas with $T_{\mathrm{k}} \simeq 150 \mathrm{~K}$ is present in NGC 253 which is compatible with our models.

The LVG models have been used for predicting the $\mathrm{CO}$ line intensities for ${ }^{12} \mathrm{CO}(1-0)$ up to ${ }^{12} \mathrm{CO}(15-14)$. We have derived the total $\mathrm{CO}$ cooling from these predictions. For Henize 2-10, we obtain with the "low $T_{\mathrm{k}}$ " model $\left(T_{\mathrm{k}}=50 \mathrm{~K}\right), 1.3 \times$ $10^{-8} \mathrm{~W} \mathrm{~m}^{-2} \mathrm{sr}^{-1}$, and with the "high $T_{\mathrm{k}}$ " model $\left(T_{\mathrm{k}}=\right.$ $100 \mathrm{~K}), 2.0 \times 10^{-8} \mathrm{~W} \mathrm{~m}^{-2} \mathrm{sr}^{-1}$. With the "low $T_{\mathrm{k}}$ " model, the lines which contribute the most to the total $\mathrm{CO}$ cooling are ${ }^{12} \mathrm{CO}(6-5)(23.4 \%)$, followed by ${ }^{12} \mathrm{CO}(5-4)(19.7 \%)$ and ${ }^{12} \mathrm{CO}(7-6)(19.5 \%)$. With the "high $T_{\mathrm{k}}$ " model, the lines which contribute the most to the total $\mathrm{CO}$ cooling are ${ }^{12} \mathrm{CO}(7-6)(19.1 \%)$ followed by ${ }^{12} \mathrm{CO}(6-5)(18.2 \%)$ and ${ }^{12} \mathrm{CO}(8-7)(15.4 \%)$.

For NGC 253, with the "low $T_{\mathrm{k}}$ " model $\left(T_{\mathrm{k}}=70 \mathrm{~K}\right)$ we deduced a total $\mathrm{CO}$ cooling of $1.0 \times 10^{-6} \mathrm{~W} \mathrm{~m}^{-2} \mathrm{sr}^{-1}$ and the most important line is ${ }^{12} \mathrm{CO}(7-6)$ (21.1\%) followed by ${ }^{12} \mathrm{CO}(6-5) \quad(20.4 \%),{ }^{12} \mathrm{CO}(8-7) \quad(15.7 \%)$ and ${ }^{12} \mathrm{CO}(5-4)(15.2 \%)$. With the "high $T_{\mathrm{k}}$ " model $\left(T_{\mathrm{k}}=\right.$ $150 \mathrm{~K}$ ), we obtained a total predicted $\mathrm{CO}$ cooling of $1.5 \times 10^{-6} \mathrm{~W} \mathrm{~m}^{-2} \mathrm{sr}^{-1}$, with the most intense lines being ${ }^{12} \mathrm{CO}(8-7)$ and ${ }^{12} \mathrm{CO}(7-6)(16.4 \%$ for each) followed by ${ }^{12} \mathrm{CO}(6-5)(13.6 \%)$ and ${ }^{12} \mathrm{CO}(9-8)(13.4 \%)$.

${ }^{12} \mathrm{CO}(6-5)$ and ${ }^{12} \mathrm{CO}(7-6)$ therefore appear to contribute significantly to the $\mathrm{CO}$ cooling. Also, CO lines with $J_{\text {upper }} \geqslant 10$ are predicted to be weak and will not have significant antenna temperatures (see Figs. 5 and 6, plots on the left side). In addition to ${ }^{12} \mathrm{CO}(6-5)$ and ${ }^{12} \mathrm{CO}(7-6)$, data for ${ }^{12} \mathrm{CO}(8-$ 7) and ${ }^{12} \mathrm{CO}(9-8)$ would be most useful in discriminating between models, and for a more accurate determination of the $\mathrm{CO}$ cooling. ${ }^{13} \mathrm{CO}(6-5)$ data would also be extremely 

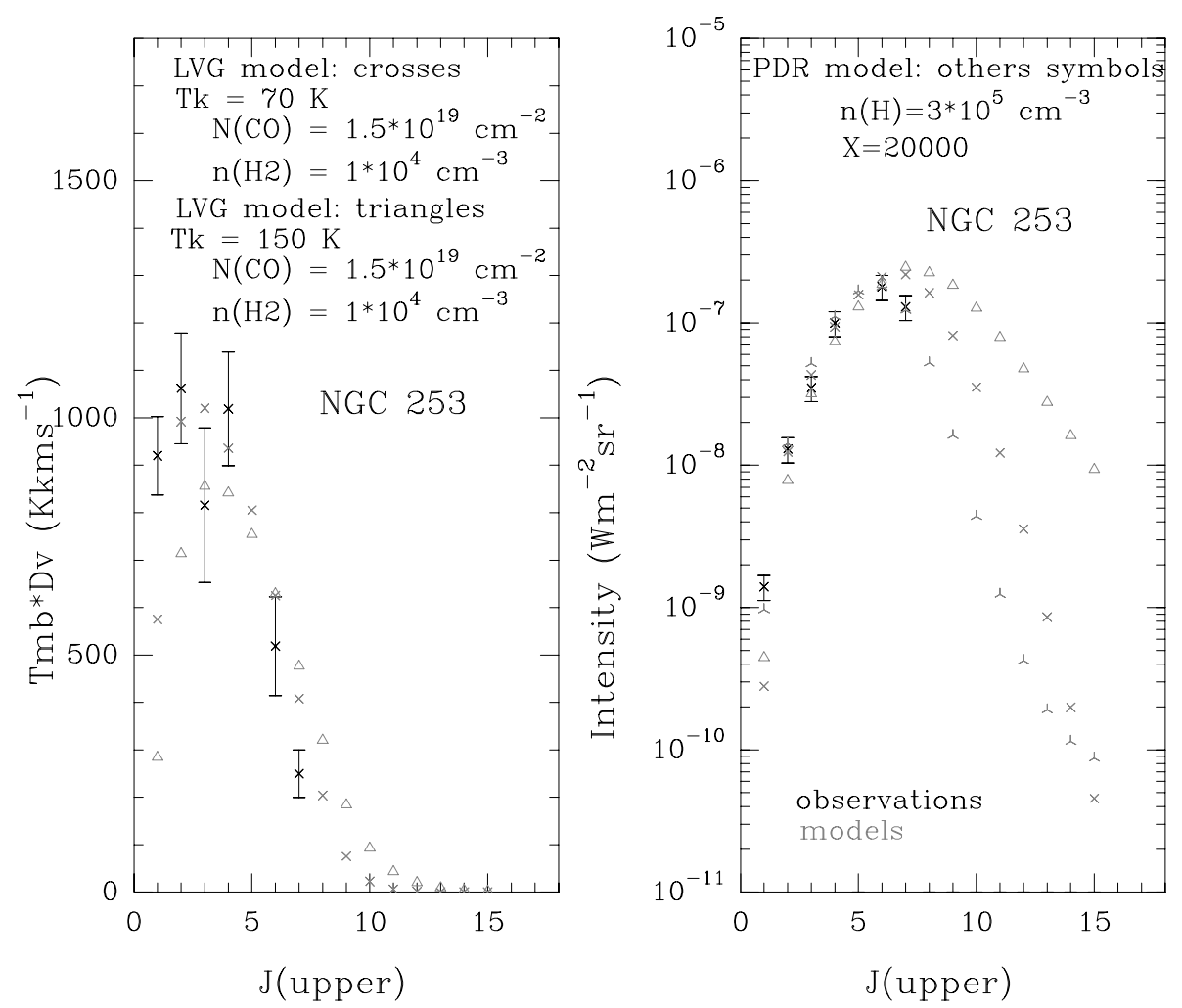

Fig. 6. LVG and PDR models calculations compared with observations for NGC 253. The observations are shown in black with error bars while model predictions are shown in grey. See caption of Fig. 5.

useful for constraining the models and for measuring the opacity of ${ }^{12} \mathrm{CO}(6-5)$ line.

\subsection{PDR model}

To progress further in the analysis of the physical conditions in the starburst nuclei, we made use of PDR models. Such models have been used here to understand the properties of the interstellar medium, since they take account of all the relevant physical and chemical processes for thermal balance. The PDR models accurately reproduce the steep kinetic temperature gradient near cloud edges, when illuminated by intense FUV radiation. Such models have been developed during the past two decades, for a variety of astrophysical sources, from giant molecular clouds illuminated by the interstellar radiation field to the conditions experienced by circumstellar disks, very close to hot massive stars (Tielens \& Hollenbach 1985a,b; Van Dishoeck \& Black 1986, 1988; Wolfire et al. 1990; Hollenbach et al. 1991; Abgrall et al. 1992; Le Bourlot et al. 1993; Köster et al. 1994; Sternberg \& Dalgarno 1995; Draine \& Bertoldi 1996; Stoerzer et al. 1996; Lhuman et al. 1997; Pak et al. 1998; Hollenbach \& Tielens 1999; Kaufman et al. 1999).

We adopted here the PDR model developed by Le Bourlot et al. (1993) for Galactic sources (see also Le Petit et al. 2002). The source is modelled as a plane-parallel slab, illuminated on both sides by FUV radiation to better reproduce the starburst environment as massive stars and giant molecular clouds are spatially correlated. Model parameters include the gas density, assumed uniform, the intensity of the illuminating FUV radiation, the gas phase elemental abundances, the grain properties, the gas to dust ratio, etc. Because the metallicities of both NGC 253 and Henize 2-10 (see Table 1) are close to solar, we used a model with Milky Way abundances $\left(12+\log \left(\frac{\mathrm{O}}{\mathrm{H}}\right)=\right.$ $8.90 \pm 0.04$ Boselli et al. 2002). We have adopted standard grain properties and gas to dust ratio appropriate for Galactic interstellar clouds. We have sampled a wide range of the parameter space, varying the gas density, $n(\mathrm{H})$ and the incident FUV flux, $\chi \times G_{0}$, where $G_{0}$ is the local average interstellar radiation field (ISRF) determined by Draine (1978) $\left(G_{0}=2.7 \times 10^{-3} \mathrm{erg} \mathrm{cm}^{-2} \mathrm{~s}^{-1}\right)$. The ${ }^{12} \mathrm{C} /{ }^{13} \mathrm{C}$ ratios $\left(X_{\text {Henize } 2-10}\right.$ and $\left.X_{\mathrm{NGC} \mathrm{253}}\right)$ are the same as for the "best" LVG models (see Table 5). Models are constrained by the ratio of integrated intensities in erg $\mathrm{cm}^{-2} \mathrm{~s}^{-1} \mathrm{sr}^{-1}$ listed in Tables 2 and 3 $\left(E\left(\mathrm{erg} \mathrm{cm}^{-2} \mathrm{~s}^{-1} \mathrm{sr}^{-1}\right)=I\left(\mathrm{~W} \mathrm{~m}^{-2} \mathrm{sr}^{-1}\right) \times 10^{3}\right)$.

Parameter pairs $(n(\mathrm{H}) ; \chi)$ which fit our dataset, are listed in Table 6. $\chi$ seems to be constrained by the ${ }^{12} \mathrm{CO}(J+$ $1 \rightarrow J) /{ }^{13} \mathrm{CO}(J+1 \rightarrow J)$ (e.g. $\left.{ }^{12} \mathrm{CO}(3-2) /{ }^{13} \mathrm{CO}(3-2)\right)$ ratios while $n(\mathrm{H})$ is more sensitive to the line ratios involving two ${ }^{12} \mathrm{CO}$ lines: ${ }^{12} \mathrm{CO}(J+1 \rightarrow J) /{ }^{12} \mathrm{CO}\left(J^{\prime}+1 \rightarrow\right.$ $\left.J^{\prime}\right)\left(\right.$ e.g. $\left.{ }^{12} \mathrm{CO}(3-2) /{ }^{12} \mathrm{CO}(6-5)\right)$ ratio. The difference between observations and model outputs is estimated to be of the order of $20 \%$. Emissivity ratios obtained from the observations and from the models are listed in Table 7.

Model predictions for the $\mathrm{CO}$ line emissivities are shown in Figs. 5 and 6 for Henize 2-10 and NGC 253 respectively. The model predictions have been scaled to match the observed line intensities. As stated above, PDR models have been 
Table 6. Parameters of the "best" PDR models.

\begin{tabular}{c|cc}
\hline \hline & Henize 2-10 & NGC 253 \\
\hline$n(\mathrm{H})\left(\mathrm{cm}^{-3}\right)$ & $8.0 \pm 1 \times 10^{5}$ & $3.0 \pm 0.5 \times 10^{5}$ \\
$\chi($ on each side $)$ & 20000 & $20000^{a}$ \\
$v^{b}\left(\mathrm{~km} \mathrm{~s}^{-1}\right)$ & 60 & 187 \\
$F F$ & $1.5 \pm 0.5 \times 10^{-3}$ & $9.7 \pm 4 \times 10^{-2}$ \\
\hline
\end{tabular}

${ }^{a}$ Carral et al. (1994) found for NGC 253 a FUV flux of $2 \times 10^{4} G_{0}$ in units of Habing (1968) corresponding to $1.2 \times 10^{4} G_{0}$ in units of Draine (1978); compatible with our value.

${ }^{b}$ Deduced from Gaussian fits to line profiles.

developed for local interstellar clouds. The velocity dispersion of the modelled cloud is a parameter in those models, which is used for computing the photo-dissociation rates of $\mathrm{H}_{2}$ and $\mathrm{CO}$, and the line emissivities. This parameter is set to $1 \mathrm{~km} \mathrm{~s}^{-1}$, a typical figure for local molecular clouds (see Wolfire et al. 1990). Using a PDR model for fitting the galaxy observations is complicated by the fact that, in a galaxy, many PDRs contribute to the signal detected in each beam, resulting in a broad line (tens to hundred of $\mathrm{km} \mathrm{s}^{-1}$ ), compared to a single PDR line $\left(1 \mathrm{~km} \mathrm{~s}^{-1}\right)$. To correct for this effect, the model line emissivities have been multiplied by the line width ratio, $\frac{\Delta v \text { (galaxy) }}{\Delta v(\mathrm{PDR})}$, where $\Delta v$ (galaxy) $\approx 190 \mathrm{~km} \mathrm{~s}^{-1}$ and $60 \mathrm{~km} \mathrm{~s}^{-1}$ for NGC 253 and Henize 2-10 respectively, and $\Delta v(\mathrm{PDR})=1 \mathrm{~km} \mathrm{~s}^{-1}$. Once this correction is performed, PDR model results are compared with observed data in the same way as the LVG models (see paragraph 5 in Sect. 5.2), and the surface filling factor of the emission in the beam, PDR_FF, is computed. The PDR_FF is $9.4 \times 10^{-2}$ for NGC 253 and $1.5 \times 10^{-3}$ for Henize 2-10. For NGC 253, the surface filling factors derived from the PDR and the LVG models are very similar. For Henize 2-10, the filling factor is significantly smaller for the PDR model than for the LVG model. In Henize 2-10, LVG models, as with PDR models, do not reproduce observations very well due to the lack of high- $J \mathrm{CO}$ lines (i.e., ${ }^{12} \mathrm{CO}(8-$ 7 ) and up) which would constrain the location of the maximum of the CO cooling curve. However, compared to LVG models, PDR models tend to fit the series of CO lines better, particularly for NGC 253. So, we prefer the PDR values of the $F F$ to those from the LVG.

We have computed the total CO cooling from PDR models summing the contribution from all $\mathrm{CO}$ lines from ${ }^{12} \mathrm{CO}(1-0)$ up to ${ }^{12} \mathrm{CO}(15-14)$. In the observations, we miss ${ }^{12} \mathrm{CO}(5-4)$ and all $\mathrm{CO}$ lines from ${ }^{12} \mathrm{CO}(8-7)$ and up. For Henize 2-10, we obtained $1.5 \times 10^{-8} \mathrm{~W} \mathrm{~m}^{-2} \mathrm{sr}^{-1}$ (similar to the $\mathrm{CO}$ cooling from the "low $T_{\mathrm{k}}$ " LVG model $\left.\left(T_{\mathrm{k}}=50 \mathrm{~K}\right)\right)$. The lines which contribute the most to the total $\mathrm{CO}$ cooling are ${ }^{12} \mathrm{CO}(7-6)(17.4 \%)$ followed by ${ }^{12} \mathrm{CO}(8-7)(15.7 \%)$ and ${ }^{12} \mathrm{CO}(6-5)$ (15.0\%) (with the "low $T_{\mathrm{k}}$ " LVG model, the lines which contribute the most are also CO lines with $J_{\text {upper }} \geqslant 5$ ). For NGC 253, we deduced a total $\mathrm{CO}$ cooling of $7.2 \times 10^{-7} \mathrm{~W} \mathrm{~m}^{-2} \mathrm{sr}^{-1}$, (close to, but lower than the $\mathrm{CO}$ cooling from the "low $T_{\mathrm{k}}$ " LVG model $\left.\left(T_{\mathrm{k}}=70 \mathrm{~K}\right)\right)$. The most important line is ${ }^{12} \mathrm{CO}(6-5)(24.9 \%)$ followed by ${ }^{12} \mathrm{CO}(5-4)(23.2 \%)$ and ${ }^{12} \mathrm{CO}(7-6)(17.0 \%)$.
Table 7. Observed and modeled line emissivity ratios for Henize 2-10 and NGC 253 using PDR models.

\begin{tabular}{|c|c|c|}
\hline $\begin{array}{c}E \\
\left(\mathrm{erg} \mathrm{cm}{ }^{-2} \mathrm{~s}^{-1} \mathrm{sr}^{-1}\right)\end{array}$ & $\begin{array}{c}\text { NGC } 253 \\
\text { observations }\end{array}$ & $\begin{array}{l}\text { Henize 2-10 } \\
\text { observations }\end{array}$ \\
\hline$\frac{{ }^{12} \mathrm{CO}(3-2)}{{ }^{12} \mathrm{CO}(6-5)}$ & $0.19 \pm 0.076^{*}$ & $0.22 \pm 0.083^{*}$ \\
\hline$\frac{{ }^{12} \mathrm{CO}(3-2)}{{ }^{12} \mathrm{CO}(7-6)}$ & $0.27 \pm 0.11^{*}$ & $0.16 \pm 0.064^{*}$ \\
\hline$\frac{{ }^{12} \mathrm{CO}(6-5)}{{ }^{12} \mathrm{CO}(7-6)}$ & $1.38 \pm 0.56^{*}$ & $0.74 \pm 0.29^{*}$ \\
\hline$\frac{{ }^{12} \mathrm{CO}(1-0)}{{ }^{13} \mathrm{CO}(1-0)}$ & $12.73 \pm 2.5^{*}$ & $16.82 \pm 6.2^{*}$ \\
\hline$\frac{{ }^{12} \mathrm{CO}(2-1)}{{ }^{13} \mathrm{CO}(2-1)}$ & $14.44 \pm 3.4^{*}$ & $22.22 \pm 6.1^{*}$ \\
\hline \multirow[t]{2}{*}{$\frac{{ }^{12} \mathrm{CO}(3-2)}{{ }^{13} \mathrm{CO}(3-2)}$} & $10.61 \pm 3.6^{*}$ & $10.21 \pm 4.7^{*}$ \\
\hline & Model & Model \\
\hline$\frac{{ }^{12} \mathrm{CO}(3-2)}{{ }^{12} \mathrm{CO}(6-5)}$ & 0.26 & 0.18 \\
\hline$\frac{{ }^{12} \mathrm{CO}(3-2)}{{ }^{12} \mathrm{CO}(7-6)}$ & 0.34 & 0.15 \\
\hline$\frac{{ }^{12} \mathrm{CO}(6-5)}{12 \mathrm{CO}(7-6)}$ & 1.32 & 0.86 \\
\hline$\frac{{ }^{12} \mathrm{CO}(1-0)}{{ }^{13} \mathrm{CO}(1-0)}$ & 15.42 & 15.07 \\
\hline$\frac{{ }^{12} \mathrm{CO}(2-1)}{{ }^{13} \mathrm{CO}(2-1)}$ & 9.72 & 8.02 \\
\hline$\frac{{ }^{12} \mathrm{CO}(3-2)}{{ }^{13} \mathrm{CO}(3-2)}$ & 7.92 & 6.27 \\
\hline
\end{tabular}

* Ratio derived from observations marked with asterisks in Tables 2 and 3 .

For NGC 253, the cooling derived from the PDR model is $\approx 40 \%$ higher than the observed CO cooling, while for Henize 2-10, the cooling from PDR models is a factor of 2 larger than the measured value. This can be explained by the fact that, for Henize 2-10, PDR models do not fit observations as well as for NGC 253. Actually, ${ }^{12} \mathrm{CO}(8-7)$ or ${ }^{12} \mathrm{CO}(9-8)$, and ${ }^{13} \mathrm{CO}(6-5)$ or ${ }^{13} \mathrm{CO}(7-6)$, would be very useful in order to determine the position of the peak of the $\mathrm{CO}$ cooling curve, and the opacity of the ${ }^{12} \mathrm{CO}(6-5)$ or ${ }^{12} \mathrm{CO}(7-6)$ lines. Finally, the strong dependence of the PDR model fit with the density $n(\mathrm{H})$, as can be seen by comparing Figs. 5 and 6, might also explain the difference between the two galaxies. We discuss this point in the next section.

\subsection{Discussion}

From the models described above, we conclude that the molecular gas in the starburst regions of Henize 2-10 and NGC 253 experience similar physical conditions: both the line ratios and derived properties are similar. Nevertheless, we can see in Figs. 5 and 6, a density difference between the two galaxies. In fact, it is difficult to fit a PDR model for Henize 2-10 since we do not have a $\mathrm{CO}$ detection for $J$ higher than $J=7-6$. The reason is the large intensity of the ${ }^{12} \mathrm{CO}(7-6)$ line relative to ${ }^{12} \mathrm{CO}(6-5)$. When the turn over in the $\mathrm{CO}$ cooling curve is not well constrained, fitting one PDR model is not easy and the solution can be understood as a "lower limit".

Moreover lower transitions of carbon monoxide like ${ }^{12} \mathrm{CO}(1-0)$ and ${ }^{12} \mathrm{CO}(2-1)$ were not really essential here since 
models were dedicated to the study of warm gas. So, the detection of high- $J$ transitions was crucial for describing the starburst nuclei.

Another point is the influence of the FWHM line width. It is obvious for NGC 253 that the $F W H M$ of the ${ }^{12} \mathrm{CO}(3-2)$ line $\left(\approx 190 \mathrm{~km} \mathrm{~s}^{-1}\right)$ is larger than that of the ${ }^{12} \mathrm{CO}(7-6)$ line $\left(\approx 95 \mathrm{~km} \mathrm{~s}^{-1}\right)$, the main reason being the better spatial resolution of the ${ }^{12} \mathrm{CO}(7-6)$ data. Because NGC 253 has a steep velocity gradient along its major axis, the convolution to lower spatial resolution, using the adopted source model, is expected to produce broader lines profiles. We tried to take into account this phenomenon in our study by using LVG models with ratios in line integrated area in $\mathrm{K} \mathrm{km} \mathrm{s}^{-1}$ instead of using ratios in main beam temperature in $\mathrm{K}$. We also studied LVG models with a smaller $\Delta v$ but this implies a lower $N(\mathrm{CO})$ since the meaningful variable in LVG models is the $\mathrm{CO}$ column density divided by the line width: $N\left({ }^{12} \mathrm{CO}\right) / \Delta v$. So different parameter pairs $\left(N\left({ }^{12} \mathrm{CO}\right), \Delta v\right)$ may reproduce the observations equally well.

PDR models predict the emissivity of the $\mathrm{CI}\left({ }^{3} \mathrm{P}_{1}-{ }^{3} \mathrm{P}_{0}\right)$ and the $\mathrm{CI}\left({ }^{3} \mathrm{P}_{2}-{ }^{3} \mathrm{P}_{1}\right)$, fine structure transitions of atomic carbon at $492 \mathrm{GHz}$ and $809 \mathrm{GHz}$ respectively (see Table 8). For Henize $2-10$, the predicted $\mathrm{CI}\left({ }^{3} \mathrm{P}_{1}-{ }^{3} \mathrm{P}_{0}\right)$ line emissivity is brighter than the observed value by a factor of 2. For NGC 253, the predicted transitions are brighter than those observed by a factor of 3 for $\mathrm{CI}\left({ }^{3} \mathrm{P}_{1}-{ }^{3} \mathrm{P}_{0}\right)$ and a factor of 12 for the $\mathrm{CI}\left({ }^{3} \mathrm{P}_{2}-{ }^{3} \mathrm{P}_{1}\right)$. We concluded that PDR models fitted to the $\mathrm{CO}$ line emission do not reproduce the atomic carbon data very well. Perhaps, to better constrain models with observed atomic carbon transitions, we should reduce the density. The finestructure lines of atomic carbon seem to share the same behaviour as the low- $J$ CO transitions. This tendancy is found in Galactic clouds also.

We compared observations of $\mathrm{CO}$ and $[\mathrm{CI}]$ in the center of the Milky Way (Fixsen et al. 1999), and in the Cloverleaf QSO (Barvainis et al. 1997; Tsuboi et al. 1999; Weiß et al. 2003) with our observations. These two objects are well known and we can compare them with NGC 253 and Henize 2-10, to determine the differences or the similarities in the physical properties of their warm gas. We chose to plot $\mathrm{CO}$ lines fluxes $\left(F\right.$ in $\left.\mathrm{W} \mathrm{m}^{-2}\right)$ versus $J_{\text {upper }}$, instead of CO lines intensities ( $I$ in $\mathrm{W} \mathrm{m}{ }^{-2} \mathrm{sr}^{-1}$ ) versus $J_{\text {upper }}$ as in Figs. 5 and 6, because the Milky Way data found in Fixsen et al. (1999) were only available in these units. The Cloverleaf QSO data (in Jy km s${ }^{-1}$ in Barvainis et al. 1997; Tsuboi et al. 1999; Weiß et al. 2003) have been converted to the same units using the Jy per $\mathrm{K}$ factors and the main beam efficiencies of telescopes (see references above). Line fluxes ( $F$ in $\mathrm{W} \mathrm{m}^{-2}$ ) are listed in Table 9 and the flux ratios in Table 10.

$\mathrm{CO}$ line fluxes $\left(F\right.$ in $\left.\mathrm{W} \mathrm{m}^{-2}\right)$ for the Cloverleaf QSO, the nucleus of the Milky Way, NGC 253 and Henize 2-10 are shown in Fig. 7. To compare the $\mathrm{CO}$ cooling curves more easily, the line fluxes have been scaled. The scaling factors are listed in the figure caption. In Fig. 7, PDR model predictions have been used for the $\mathrm{CO}$ lines which are lacking observations: ${ }^{12} \mathrm{CO}(5-4)$ and ${ }^{12} \mathrm{CO}(8-7)$ for NGC 253 and for Henize 2-10 (see Table 9). Note that the PDR model used for Henize 2-10 PDR does not reproduce observations as well as the PDR model used for NGC 253 (see Figs. 5 and 6).
Table 8. Results of PDR models for [CI] in Henize 2-10 and NGC 253. We reported also observations from Tables 2 and 3. The PDR model predictions have been multiplied by the line width ratio, $\frac{\Delta v(\text { galaxy })}{\Delta v(\mathrm{PDR})}$, and by the surface filling factor, PDR_FF, (see Table 6).

\begin{tabular}{c|cc}
\hline \hline $\begin{array}{c}E \\
\left(\mathrm{erg} \mathrm{cm}^{-2} \mathrm{~s}^{-1} \mathrm{sr}^{-1}\right)\end{array}$ & $\begin{array}{c}\text { NGC 253 } \\
\text { observations }\end{array}$ & $\begin{array}{c}\text { Henize 2-10 } \\
\text { observations }\end{array}$ \\
\hline $\mathrm{CI}\left({ }^{3} \mathrm{P}_{1}-{ }^{3} \mathrm{P}_{0}\right)$ & $3.5 \times 10^{-5}$ & $3.0 \times 10^{-7}$ \\
$\mathrm{CI}\left({ }^{3} \mathrm{P}_{2}-{ }^{3} \mathrm{P}_{1}\right)$ & $3.2 \times 10^{-5}$ & Not observed \\
\hline & PDR model & PDR model \\
\hline $\mathrm{CI}\left({ }^{3} \mathrm{P}_{1}-{ }^{3} \mathrm{P}_{0}\right)$ & $1.1 \times 10^{-4}$ & $5.1 \times 10^{-7}$ \\
$\mathrm{CI}\left({ }^{3} \mathrm{P}_{2}-{ }^{3} \mathrm{P}_{1}\right)$ & $3.9 \times 10^{-4}$ & $1.9 \times 10^{-6}$ \\
\hline
\end{tabular}

We compare the CO cooling curve for the center of the Milky Way, the Cloverleaf QSO, Henize 2-10 and NGC 253. The turnover positions for Henize 2-10, NGC 253 and the Cloverleaf QSO are close to each other, near ${ }^{12} \mathrm{CO}(6-5)$ or above, while the turnover of the $\mathrm{CO}$ cooling curve of the nucleus of the Milky Way is near ${ }^{12} \mathrm{CO}(4-3)$. We may ask if this observed difference between the Milky Way and the three other sources is consistent with a difference in linear resolution, or if it is due to differences in inherent physical conditions between the four environments. The linear resolution for NGC 253 corresponding to the adopted beam size of $21.9^{\prime \prime}$ is $265 \mathrm{pc}$. For Henize 2-10, with a distance of $6 \mathrm{Mpc}$ and the same beam size, we obtained a linear resolution of $637 \mathrm{pc}$. For the Cloverleaf QSO, we computed the angular distance from $H_{0}$ and $q_{0}$ in Weiß et al. (2003), we obtained $D_{A}=1056.4 \mathrm{Mpc}$ and a linear resolution of $13 \mathrm{kpc}$. For the nucleus of the Milky Way, the $7^{\circ}$ COBE-FIRAS beam gives a linear resolution of $1035 \mathrm{pc}$ in the Galactic Center (distance of $8.5 \mathrm{kpc}$ ). We conclude that despite the wide range of linear resolutions, the turnover of the $\mathrm{CO}$ cooling curve is found at roughly the same $J$ value, above ${ }^{12} \mathrm{CO}(6-5)$ for Henize 2-10, NGC 253 and the Cloverleaf QSO. However, at the same linear scale as Henize 2-10, the gas in the Milky Way nucleus shows less excitation. Thus, it seems likely that differences in the shapes of the $\mathrm{CO}$ cooling curves are due to differences in the ISM properties in the target galaxies. The fact that the Cloverleaf QSO, NGC 253 and Henize 2-10 have the same ratios is related to the similarity of the physical properties of warm gas for these three sources, which translates into similar CO line ratios (see Table 10).

\section{Conclusions}

We observed Henize 2-10 in the rotational lines of carbon monoxide ${ }^{12} \mathrm{CO}(J=3-2),(J=4-3),(J=6-5)$ and $(J=7-6)$ and NGC 253 in the ${ }^{3} \mathrm{P}_{2}-{ }^{3} \mathrm{P}_{1}$ fine structure transitions of atomic carbon $[\mathrm{CI}]$ at $809 \mathrm{GHz}$ and in the rotational lines of carbon monoxide ${ }^{12} \mathrm{CO}(3-2),(J=6-5)$ and $(J=7-6)$. We show that $\mathrm{C}$ cooling is less than $\mathrm{CO}$ cooling for both galaxy nuclei by a factor $>10$. Among observed lines, those which contribute most to the $\mathrm{CO}$ cooling are ${ }^{12} \mathrm{CO}(6-5)$ and ${ }^{12} \mathrm{CO}(7-6)$.

Such high- $J$ transitions are needed to constrain the physical conditions in starburst nuclei. We used both LVG and PDR models for each galaxy: the molecular gas in the 
Table 9. Observations of the Milky Way nucleus (from Fixsen et al. 1999), NGC 253 (from this paper), Henize 2-10 (from this paper) and the Cloverleaf QSO (from Barvainis et al. 1997; Tsuboi et al. 1999; Weiß et al. 2003).

\begin{tabular}{c|cccc}
\hline \hline $\begin{array}{c}F \\
\left(\mathrm{~W} \mathrm{~m}^{-2}\right)\end{array}$ & Milky Way & NGC 253 & Henize 2-10 & Cloverleaf \\
\hline $\mathrm{CI}\left({ }^{3} \mathrm{P}_{1}-^{3} \mathrm{P}_{0}\right)$ & $1.9 \times 10^{-11}$ & $4.6 \times 10^{-16}$ & $3.8 \times 10^{-18}$ & $1.7 \times 10^{-23}$ \\
$\mathrm{CI}\left({ }^{3} \mathrm{P}_{2}-{ }^{3} \mathrm{P}_{1}\right)$ & $1.9 \times 10^{-11}$ & $4.0 \times 10^{-16}$ & Not observed & $3.9 \times 10^{-23}$ \\
\hline${ }^{12} \mathrm{CO}(1-0)$ & $2.7 \times 10^{-12}$ & $2.0 \times 10^{-17}$ & $4.8 \times 10^{-19}$ & $\leq 1.5 \times 10^{-24}$ \\
${ }^{12} \mathrm{CO}(2-1)$ & $1.1 \times 10^{-11}$ & $1.9 \times 10^{-16}$ & $2.5 \times 10^{-18}$ & \\
${ }^{12} \mathrm{CO}(3-2)$ & $2.0 \times 10^{-11}$ & $4.4 \times 10^{-16}$ & $9.0 \times 10^{-18 a}$ & $4.3 \times 10^{-23}$ \\
${ }^{12} \mathrm{CO}(4-3)$ & $3.0 \times 10^{-11}$ & $1.3 \times 10^{-15}$ & $1.4 \times 10^{-17}$ & $9.1 \times 10^{-23}$ \\
${ }^{12} \mathrm{CO}(5-4)$ & $2.8 \times 10^{-11}$ & $2.2 \times 10^{-15 b}$ & $3.3 \times 10^{-17 b}$ & $1.3 \times 10^{-22}$ \\
${ }^{12} \mathrm{CO}(6-5)$ & $2.0 \times 10^{-11}$ & $2.2 \times 10^{-15}$ & $2.9 \times 10^{-17}$ & \\
${ }^{12} \mathrm{CO}(7-6)$ & $1.7 \times 10^{-11}$ & $1.7 \times 10^{-15}$ & $4.0 \times 10^{-17}$ & $3.6 \times 10^{-22}$ \\
${ }^{12} \mathrm{CO}(8-7)$ & $1.9 \times 10^{-11}$ & $6.7 \times 10^{-16 b}$ & $4.9 \times 10^{-17 b}$ & \\
\hline
\end{tabular}

${ }^{a}$ We chose to use ${ }^{12} \mathrm{CO}(3-2)$ observations from Meier et al. (2001) because they seem to agree with LVG and PDR models (see Fig. 5) better than our observations.

${ }^{b}$ These values are computed from PDR models.

Table 10. Flux ratios for the center of the Milky Way, NGC 253, Henize 2-10 and the Cloverleaf QSO.

\begin{tabular}{c|cccc}
\hline \hline $\begin{array}{c}F \\
\left(\mathrm{~W} \mathrm{~m}{ }^{-2}\right)\end{array}$ & Milky Way & NGC 253 & Henize 2-10 & Cloverleaf \\
\hline$\frac{\mathrm{CI}\left({ }^{3} \mathrm{P}_{1}{ }^{3} \mathrm{P}_{0}\right)}{\mathrm{CI}^{\left(\mathrm{P}_{2}{ }^{3} \mathrm{P}_{1}\right)}}$ & 1.00 & 1.15 & Not observed & 0.42 \\
$\frac{12 \mathrm{CO}(3-2)}{12 \mathrm{CO}(1-0)}$ & 7.37 & 22 & 18.75 & $\geq 28.6$ \\
$\frac{{ }^{12} \mathrm{CO}(3-2)}{12 \mathrm{CO}(2-1)}$ & 1.84 & 2.32 & 3.6 & - \\
$\frac{12 \mathrm{CO}(3-2)}{12 \mathrm{CO}(4-3)}$ & 0.67 & 0.34 & 0.64 & 0.47 \\
$\frac{12 \mathrm{CO}(3-2)}{12 \mathrm{CO}(5-4)}$ & 0.72 & $0.21^{*}$ & $0.27^{*}$ & 0.33 \\
$\frac{12 \mathrm{CO}(3-2)}{12 \mathrm{CO}(6-5)}$ & 1.03 & 0.20 & 0.31 & - \\
$\frac{12 \mathrm{CO}(3-2)}{12 \mathrm{CO}(7-6)}$ & 1.18 & 0.26 & 0.23 & 0.12 \\
$\frac{12 \mathrm{CO}(3-2)}{12 \mathrm{CO}(8-7)}$ & 1.09 & $0.66^{*}$ & $0.18^{*}$ & - \\
\hline
\end{tabular}

* These values are computed from PDR models.

Henize 2-10 nucleus is well described with a LVG model defined by $\Delta V=60 \mathrm{~km} \mathrm{~s}^{-1}, T_{\mathrm{k}} \approx 50-100 \mathrm{~K},{ }^{12} \mathrm{CO} \approx 30$, $n\left(\mathrm{H}_{2}\right) \gtrsim 10^{4} \mathrm{~cm}^{-3}$ and $N\left({ }^{12} \mathrm{CO}\right)=3.5 \pm 1 \times 10^{18} \mathrm{~cm}^{-2}$. For the NGC 253 nucleus, we derived the following parameters: $\Delta V=$ $190 \mathrm{~km} \mathrm{~s}^{-1}, T_{\mathrm{k}} \approx 70-150 \mathrm{~K}, \frac{{ }^{12} \mathrm{CO}}{{ }^{13} \mathrm{CO}} \approx 40, n\left(\mathrm{H}_{2}\right) \approx 10^{4} \mathrm{~cm}^{-3}$ and $N\left({ }^{12} \mathrm{CO}\right)=1.5 \pm 0.5 \times 10^{19} \mathrm{~cm}^{-2}$. PDR models provide equally good fits to the data. PDR models were used to give us more information about the physical parameters in these media. We succeeded in reproducing the observations with an accuracy of about $20 \%$ using a PDR model defined for Henize 2-10 by $n(\mathrm{H})=8.0 \pm 1 \times 10^{5} \mathrm{~cm}^{-3}$ and $\chi=20000$ (we modelled the source as a plane-parallel slab, illuminated on both sides by FUV radiation). For NGC 253, we derived the following model: $n(\mathrm{H})=3.0 \pm 0.5 \times 10^{5} \mathrm{~cm}^{-3}$ and $\chi=20000$.
Thanks to those models, we predict that, for distant galaxies, to obtain properties of cold and warm gas, the most interesting $\mathrm{CO}$ lines to be observed are ${ }^{12} \mathrm{CO}(1-0),(2-1),(3-2),(4-3)$, (5-4), (6-5), (7-6) and ${ }^{12} \mathrm{CO}(8-7) .{ }^{12} \mathrm{CO}(9-8)$ and higher- $J$ $\mathrm{CO}$ lines will be weaker and more difficult to detect in distant galaxies. The reasons are twofold. First the most intense lines are ${ }^{12} \mathrm{CO}(6-5)$ and ${ }^{12} \mathrm{CO}(7-6)$, second, though ${ }^{12} \mathrm{CO}(9-8)$ can have a significant contribution to the cooling, its antenna temperature is significantly lower than for other $\mathrm{CO}$ lines (e.g. ${ }^{12} \mathrm{CO}(4-3)$ or $\left.{ }^{12} \mathrm{CO}(6-5)\right)$. Also, the typical $T_{\mathrm{k}}$ and $n\left(\mathrm{H}_{2}\right)$ values only allow significant excitation over a large fraction of the galaxy for $J_{\text {up }} \leqslant 10$ transitions. For higher- $J$ transitions the values of $T_{\mathrm{k}}$ and $n\left(\mathrm{H}_{2}\right)$ high enough to excite $J_{\text {up }} \geqslant 10$ will be confined to a volume too small to produce a detectable signal. 


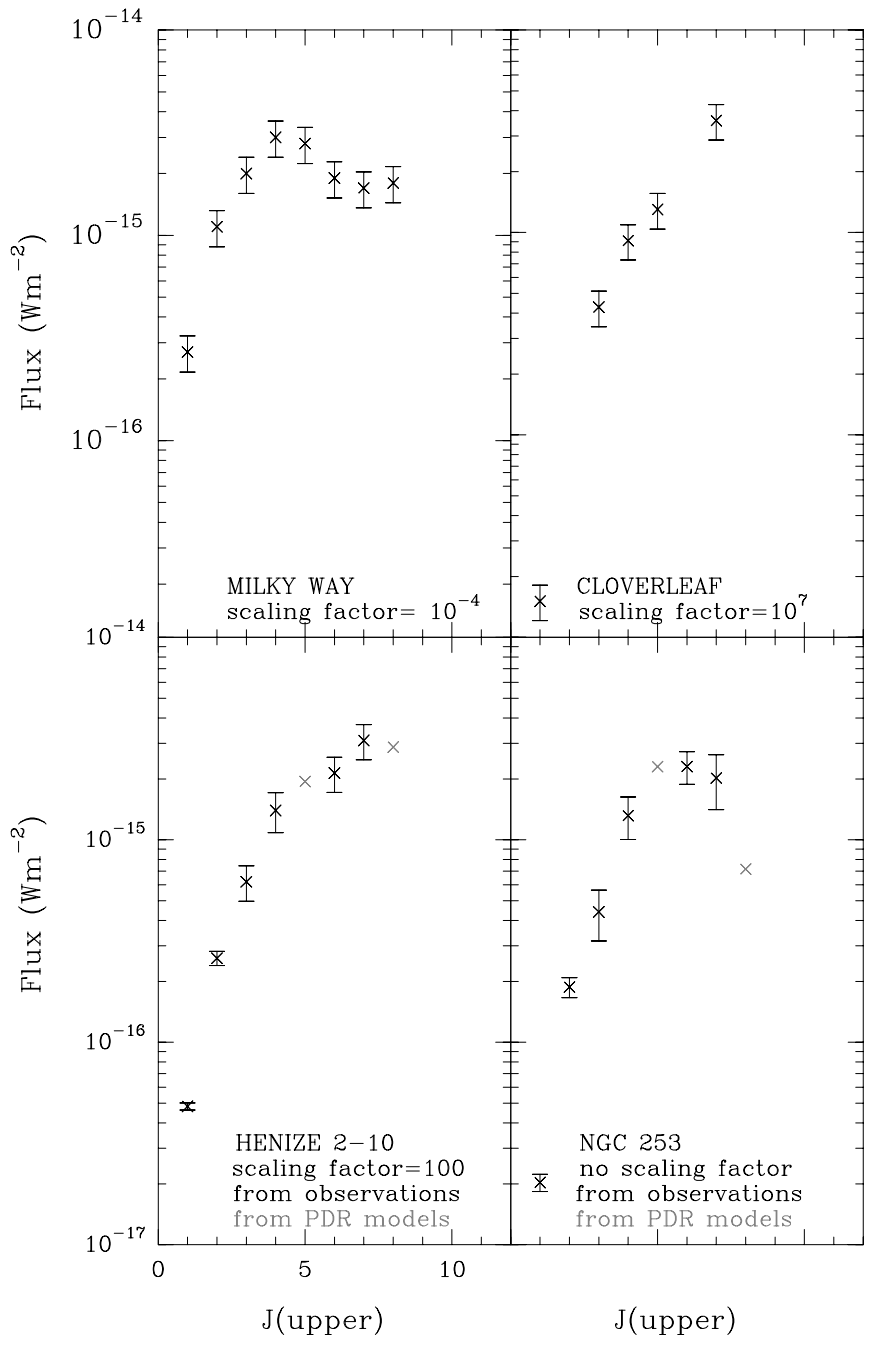

Fig. 7. Flux ( $\mathrm{W} \mathrm{m}^{-2}$ ) vs. $J_{\text {upper }}$ for the nucleus of the Milky Way (top left), NGC 253 (top right), Henize 2-10 (bottom left) and the Cloverleaf QSO (bottom right). PDR models have been used to obtained fluxes (plotted in grey) of ${ }^{12} \mathrm{CO}(5-4)$ and ${ }^{12} \mathrm{CO}(8-7)$ in Henize 2-10 and in NGC 253. Observations are shown in black. To facilitate the comparison, we applied scaling factors of $1 \times 10^{7}$ for the Cloverleaf QSO data, $1 \times 10^{-4}$ for the nucleus of the Milky Way, and 100 for Henize 2-10. Data for NGC 253 are shown with no scaling factor. For the Cloverleaf QSO, the ${ }^{12} \mathrm{CO}(1-0)$ line flux is an upper limit (see Tsuboi et al. 1999).

Data on the ${ }^{12} \mathrm{CO}(8-7)$ and on the ${ }^{13} \mathrm{CO}(6-5)$ lines would be extremely useful in further studies; ${ }^{12} \mathrm{CO}(8-7)$ will help to localize the maximum of the $\mathrm{CO}$ cooling curve, while ${ }^{13} \mathrm{CO}(6-5)$ will help constrain the warm gas column density. Even now, it is still difficult to detect these lines from the ground; with APEX receivers which may cover atmospheric windows up to a frequency of $1.4 \mathrm{THz}^{2}$, these lines could become available. ALMA will give access to better spatial resolution, for resolving individual molecular clouds. And with HIFI and PACS on board the Herschel satellite, lines with frequencies up to $5 \mathrm{THz}$ will become accessible.

\footnotetext{
${ }^{2}$ http://www.mpifr-bonn.mpg.de/div/mm/apex.html
}

We compared properties of warm gas derived from our models with the properties of the nucleus of the Milky Way and of the Cloverleaf QSO. We concluded that the ISM in NGC 253, Henize 2-10 and in the Cloverleaf QSO are similar, leading to similar CO excitation, while the nucleus of the Milky Way exhibits lower excitation CO lines.

Acknowledgements. This work has benefitted from financial support from CNRS-PCMI and CNRS-INSU travel grants. We thank J. Cernicharo for letting us use his CO LVG model and J. Le Bourlot et P. Hily-Blant for introducing us to his PDR model. The CSO is funded by the NSF under contract \# AST 9980846. We thanks the referee for the useful comments.

\section{References}

Abgrall, H., Le Bourlot, J., Pineau Des Forets, G., et al. 1992, A\&A, 253,525

Allen, D. A., Wright, A. E., \& Goss, W. M. 1976, MNRAS, 177, 91

Antonucci, R. R. J., \& Ulvestad, J. S. 1988, ApJ, 330, L97

Arimoto, N., Sofue, Y., \& Tsujimoto, T. 1996, PASJ, 48, 275

Baas, F., Israel, F. P., \& Koornneef, J. 1994, A\&A, 284, 403

Barvainis, R., Maloney, P., Antonucci, R., \& Alloin, D. 1997, ApJ, 484, 695

Bennett, C. L., Fixsen, D. J., Hinshaw, G., et al. 1994, ApJ, 434, 587

Bertoldi, F., Cox, P., Neri, R., et al. 2003, A\&A, 409, L47

Blecha, A. 1986, A\&A, 154, 321

Boselli, A., Lequeux, J., \& Gavazzi, G. 2002, A\&A, 384, 33

Bradford, C. M., Nikola, T., Stacey, G. J., et al. 2003, ApJ, 586, 891

Carlstrom, J. E., Jackson, J. M., Ho, P. T. P., \& Turner, J. L. 1990, NASA, Ames Research Center, The Interstellar Medium in External Galaxies: Summaries of Contributed Papers (SEE N9114100 05-90), 337

Carral, P., Hollenbach, D. J., Lord, S. D., et al. 1994, ApJ, 423, 223

Cox, P., Omont, A., Djorgovski, S. G., et al. 2002, A\&A, 387, 406

Davidge, T. J., \& Pritchet, C. J. 1990, AJ, 100, 102

De Jong, T., Dalgarno, A., \& Chu, S.-I. 1975, ApJ, 199, 69

D’Odorico, S., Rosa, M., \& Wampler, E. J. 1983, A\&AS, 53, 97

Draine, B. T. 1978, ApJS, 36, 595

Draine, B. T., \& Bertoldi, F. 1996, ApJ, 468, 269

Fixsen, D. J., Bennett, C. L., \& Mather, J. C. 1999, ApJ, 526, 207

Frerking, M. A., Langer, W. D., \& Wilson, R. W. 1982, ApJ, 262, 590

Gerin, M., \& Phillips, T. G. 1998, ApJ, 509, L17

Gerin, M., \& Phillips, T. G. 2000, ApJ, 537, 644

Goldreich, P., \& Kwan, J. 1974, ApJ, 189, 441

Goldsmith, P. F. 2001, ApJ, 557, 736

Goldsmith, P. F., \& Langer, W. D. 1978, ApJ, 222, 881

Guesten, R., Serabyn, E., Kasemann, C., et al. 1993, ApJ, 402, 537

Habing, H. J. 1968, Bull. Astron. Inst. Netherlands, 19, 421

Harris, A. I., Stutzki, J., Graf, U. U., et al. 1991, ApJ, 382, L75

Harrison, A., Puxley, P., Russell, A., \& Brand, P. 1995, MNRAS, 277, 413

Harrison, A., Henkel, C., \& Russell, A. 1999, MNRAS, 303, 157

Henkel, C., \& Mauersberger, R. 1993, A\&A, 274, 730

Henkel, C., Mauersberger, R., Wiklind, T., et al. 1993, A\&A, 268, L17

Hollenbach, D. J., \& Tielens, A. G. G. M. 1999, Rev. Mod. Phys., 71, 173

Hollenbach, D. J., Takahashi, T., \& Tielens, A. G. G. M. 1991, ApJ, 377, 192

Imanishi, M., \& Dudley, C. C. 2000, ApJ, 545, 701

Israel, F. P., \& Baas, F. 2001, A\&A, 371, 433

Israel, F. P., \& Baas, F. 2002, A\&A, 383, 82 
Israel, F. P., \& Baas, F. 2003, A\&A, 404, 495

Israel, F. P., White, G. J., \& Baas, F. 1995, A\&A, 302, 343

Johansson, I. 1987, A\&A, 182, 179

Kaufman, M. J., Wolfire, M. G., Hollenbach, D. J., \& Luhman, M. L. 1999, ApJ, 527, 795

Kawara, K., Nishida, M., Taniguchi, Y., \& Jugaku, J. 1987, PASP, 99, 512

Keto, E., Hora, J. L., Fazio, G. G., Hoffmann, W., \& Deutsch, L. 1999, ApJ, 518, 183

Kobulnicky, H. A., Dickey, J. M., Sargent, A. I., Hogg, D. E., \& Conti, P. S. 1995, AJ, 110, 116

Kobulnicky, H. A., Kennicutt, R. C., \& Pizagno, J. L. 1999, ApJ, 514, 544

Koester, B., Stoerzer, H., Stutzki, J., \& Sternberg, A. 1994, A\&A, 284, 545

Kutner, M. L., \& Ulich, B. L. 1981, ApJ, 250, 341

Lagache, G., Dole, H., \& Puget, J.-L. 2003, MNRAS, 338, 555

Le Bourlot, J., Pineau des Forets, G., Roueff, E., \& Schilke, P. 1993, ApJ, 416, L87

Le Petit, F., Roueff, E., \& Le Bourlot, J. 2002, A\&A, 390, 369

Luhman, M. L., Jaffe, D. T., Sternberg, A., Herrmann, F., \& Poglitsch, A. 1997, ApJ, 482, 298

Mauersberger, R., Henkel, C., Wielebinski, R., Wiklind, T., \& Reuter, H.-P. 1996, A\&A, 305, 421

Meier, D. S., Turner, J. L., Crosthwaite, L. P., \& Beck, S. C. 2001, AJ, 121,740

Mirabel, I. F., Vigroux, L., Charmandaris, V., et al. 1998, A\&A, 333, L1

Paglione, T. A. D., Yam, O., Tosaki, T., \& Jackson, J. M. 2004, ApJ, 611,835

Pak, S., Jaffe, D. T., van Dishoeck, E. F., Johansson, L. E. B., \& Booth, R. S. 1998, ApJ, 498, 735

Pence, W. D. 1980, ApJ, 239, 54
Peng, R., Zhou, S., Whiteoak, J. B., Lo, K. Y., \& Sutton, E. C. 1996, ApJ, 470, 821

Rieke, G. H., Lebofsky, M. J., \& Walker, C. E. 1988, ApJ, 325, 679

Rigopoulou, D., Kunze, D., Lutz, D., Genzel, R., \& Moorwood, A. F. M. 2002, A\&A, 389, 374

Sanders, D. B., \& Mirabel, I. F. 1996, ARA\&A, 34, 749

Sorai, K., Nakai, N., Kuno, N., Nishiyama, K., \& Hasegawa, T. 2000, PASJ, 52, 785

Sternberg, A., \& Dalgarno, A. 1995, ApJS, 99, 565

Stoerzer, H., Stutzki, J., \& Sternberg, A. 1996, A\&A, 310, 592

Strickland, D. K., Heckman, T. M., Colbert, E. J. M., Hoopes, C. G., \& Weaver, K. A. 2004, ApJS, 151, 193

Stutzki, J., \& Guesten, R. 1990, ApJ, 356, 513

Telesco, C. M., \& Harper, D. A. 1980, ApJ, 235, 392

Tielens, A. G. G. M., \& Hollenbach, D. 1985a, ApJ, 291, 747

Tielens, A. G. G. M., \& Hollenbach, D. 1985b, ApJ, 291, 722

Tsuboi, M., Miyazaki, A., Imaizumi, S., \& Nakai, N. 1999, PASJ, 51, 479

Turner, B. E. 1985, ApJ, 299, 312

van Dishoeck, E. F., \& Black, J. H. 1986, ApJS, 62, 109

van Dishoeck, E. F., \& Black, J. H. 1988, ApJ, 334, 771

Wall, W. F., Jaffe, D. T., Bash, F. N., \& Israel, F. P. 1991, ApJ, 380, 384

Walter, F., Bertoldi, F., Carilli, C., et al. 2003, Nature, 424, 406

Ward, J. S., Zmuidzinas, J., Harris, A. I., \& Isaak, K. G. 2003, ApJ, 587,171

Weiß, A., Henkel, C., Downes, D., \& Walter, F. 2003, A\&A, 409, L41

Wilson, C. D. 1995, ApJ, 448, L97

Wolfire, M. G., Tielens, A. G. G. M., \& Hollenbach, D. 1990, ApJ, 358,116

Yun, M. S., \& Carilli, C. L. 2002, ApJ, 568, 88

Zaritsky, D., Kennicutt, R. C., \& Huchra, J. P. 1994, ApJ, 420, 87 